\title{
A Survey of Techniques and Challenges in Underwater Localization
}

\author{
Hwee-Pink Tan ${ }^{\mathrm{a}, *}$, Roee Diamant ${ }^{\mathrm{b}}$, Winston K.G. Seah ${ }^{\mathrm{c}, * *}$, Marc \\ Waldmeyer ${ }^{\mathrm{d}, 1}$ \\ ${ }^{a}$ Networking Protocols Department, Institute for Infocomm Research (Singapore) \\ ${ }^{b}$ Department of Electrical and Computer Engineering, The University of British \\ Columbia (Canada) \\ ${ }^{c}$ School of Engineering and Computer Science, Victoria University of Wellington, \\ Wellington (New Zealand) \\ ${ }^{d}$ Laboratory for Computer Communications and Applications, EPFL (Switzerland)
}

\begin{abstract}
Underwater Wireless Sensor Networks (UWSNs) are expected to support a variety of civilian and military applications. Sensed data can only be interpreted meaningfully when referenced to the location of the sensor, making localization an important problem. While Global Positioning System (GPS) receivers are commonly used in terrestrial WSNs to achieve this, this is infeasible in UWSNs as GPS signals do not propagate through water. Acoustic communications is the most promising mode of communication underwater. However, underwater acoustic channels are characterized by harsh physical layer conditions with low bandwidth, high propagation delay and high bit er-
\end{abstract}

\footnotetext{
*Principal corresponding author; Tel: +65 64082274; Fax: +65 67761378

${ }^{* *}$ Corresponding author

Email addresses: hptan@i2r.a-star.edu.sg (Hwee-Pink Tan), roeed@ece.ubc.ca (Roee Diamant), Winston.Seah@ecs.vuw.ac.nz (Winston K.G. Seah), marc.waldmeyer@epfl.ch (Marc Waldmeyer)

${ }^{1}$ This author was involved in this work as a postgraduate intern at the Institute for Infocomm Research from September 2009 to March 2010.
} 
ror rate. Moreover, the variable speed of sound, and the non-negligible node mobility due to water currents pose a unique set of challenges for localization in UWSNs. In this paper, we provide a survey of techniques and challenges in localization specifically for UWSNs. We categorize them into (i) range-based vs range-free techniques; (ii) techniques that rely on static reference nodes vs. those who also rely on mobile reference nodes, and (iii) single-stage vs. multi-stage schemes. We compare the schemes in terms of localization speed, accuracy, coverage and communication costs. Finally, we provide an outlook on the challenges that should be, but have yet been, addressed.

Keywords: underwater localization, acoustic communications, underwater sensor networks

\section{Introduction}

During the last couple of years, we could observe a growing interest in Underwater Wireless Sensor Networks (UWSNs). One important reason is that they can improve ocean exploration and fulfil the needs of a multitude of underwater applications, including: oceanographic data collection, warning systems for natural disasters (e.g., seismic and tsunami monitoring), ecological applications (e.g., pollution, water quality and biological monitoring), military underwater surveillance, assisted navigation, industrial applications (offshore exploration), etc. For example, in offshore engineering applications, the sensors can measure parameters such as foundation strength and mooring tensions to monitor the structural health of deepwater mooring systems.

Two common communications architecture for UWSNs are shown in Figure 1 . In addition to underwater sensor nodes, the network may also comprise 
surface stations and autonomous underwater vehicles (AUVs). Regardless of the type of deployment (outdoor, indoor, underground or underwater), the location of the sensors needs to be determined for meaningful interpretation of the sensed data. Since RF communications are significantly attenuated underwater (Burdic, 2002), the use of the well-known Global Positioning System (GPS) is restricted to surface nodes. Hence, message exchanges between submerged UWSN nodes and surface nodes (or other reference nodes with known

locations) needed for localization must be carried out, usually using acoustic communications. Unfortunately, underwater acoustic channels are characterized by long propagation delays, limited bandwidth, motion-induced Doppler shift, phase and amplitude fluctuations, multipath interference, etc (Burdic, 2002). These characteristics pose severe challenges towards designing localization schemes that fulfil the following desirable properties:

\section{- High Accuracy}

The location of the sensor for which sensed data is derived should be accurate and unambiguous for meaningful interpretation of data. Localization protocols usually minimizes the distance between the estimated and true locations.

\section{- Fast Convergence}

Since nodes may drift due to water currents, the localization procedure should be fast so that it reports the actual location when data is sensed.

\section{- Wide Coverage}

The localization scheme should ensure that most of the nodes in the network can be localized. 


\section{- Low Communication Costs}

Since the nodes are battery-powered and may be deployed for long durations, communication overhead should be minimized.

\section{- Good Scalability}

The long propagation delay and relatively high power attenuation in the underwater acoustic channel pose a scalability problem where performance is highly affected by the number of nodes in the network. Consequently, an underwater acoustic localization protocol should be distributed and rely on as few reference nodes as possible.

In addition to the above quantifiable properties, practical considerations such as ease and cost of deploying reference nodes and other required infrastructure should be taken into account too.

In general, localization schemes in terrestrial wireless sensor networks can be classified into three categories: geometric analysis approach, proximity approach and scene analysis approach (Hightower and Borriello, 2001). With geometric analysis or range-based approaches, each ordinary node (node to be localized) relies on time and/or bearing information to evaluate its distance to other reference nodes (with known locations) in the system. It then utilizes multilateration/angulation to estimate its own location. On the other hand, in proximity approaches, ordinary nodes infer their proximity to reference nodes (e.g., in terms of number of hops) so as to achieve coarse localization, e.g., in an area instead of a specific location. Last but not least, scene analysis obtains localization information by analyzing "pictures" taken by the sensor nodes and comparing the pictures with previously available "training" data. 
Although localization has been widely studied for terrestrial wireless sensor networks, existing techniques cannot be directly applied to UWSNs due to the challenges associated with such networks. In a previous survey (Chandrasekhar et al., 2006), the authors explore such schemes for UWSNs, as well as the challenges in meeting the requirements posed by UWSNs for offshore engineering applications. Since then, a multitude of localization schemes have been proposed specifically for UWSNs.

In this paper, we present a survey of these schemes by further categorizing them into schemes that rely solely on static references vs those that employ mobile references, and single-stage vs. multi-stage schemes: ordinary nodes do not become "new" reference nodes to help localize other ordinary nodes in single-stage schemes, but do so in multi-stage schemes.

We also describe the salient features of key schemes and provide a qualitative evaluation in terms of speed, accuracy, coverage and communication costs. In addition, we also identify important challenges that should be addressed, and discuss the extent to which they have been addressed by existing schemes. The classification of each localization scheme surveyed in this paper is shown in Figure 2.

We complement our survey with a discussion of commercially available underwater positioning systems, where we describe the state-of-the-art as well as the precision of these systems.

This paper is organized as follows: In Section 2, since most underwater localization schemes are range-based, we first classify these schemes, identify the challenges and describe how and to what extent they are met. Next, in Section 3, we describe the salient features of range-free schemes. Following 
this, we describe the state-of-the-art as well as the precision of commercially available systems for underwater positioning in Section 4. Finally, we provide a summary in Section 5, and outline some open problems and research challenges to be addressed in Section 6 .

\section{Range-based Underwater Localization}

Range-based localization typically comprises the following steps:

- Step 1a: Range measurement (Reference node within communication range of ordinary node)

Each ordinary node estimates its distance from each reference node using the following methods:

\section{- Received Signal Strength Indicator (RSSI)}

Each ordinary node determines its distance from a reference node by measuring the Received Signal Strength and comparing it with a range dependent signal attenuation model. However, it is difficult to achieve accurate ranging when multipath and shadow fading effects exist (Burdic, 2002). Since the path loss in underwater acoustic channels is usually time varying and multipath effect can result in significant energy fading, the RSSI method is not the primary choice for underwater localization.

\section{- Time Difference of Arrival (TDoA)}

For indoor localization, the TDoA method utilizes the time difference between two different transmission mediums, namely, radio transmission and acoustic transmission, to calculate the distance 
between objects (Gu et al., 2006). Based on the two received signals, the distance to the transmitter can be determined. However, it is unsuitable for underwater localization because radio does not propagate well in water. Alternatively, the time difference of arrival between beacons from different reference nodes transmitted using acoustic signalling can be used in localization, e.g., in Cheng et al. (2008).

\section{- Time of Arrival (ToA)}

The Time of Arrival (ToA) method performs ranging based on the relationship among transmission time, speed and distance. Most proposed range-based localization schemes use this method due to the limitations of the RSSI and TDoA-based approaches. However, ToA techniques may require time synchronization between network nodes.

\section{- Step 1b: Range measurement (Reference node outside com-} munication range of ordinary node)

In this case, each ordinary node estimates its distance from each reference node using techniques such as Euclidean distance propagation method (Niculescu and Nathi, 2001).

\section{- Step 2: Location estimation}

Each ordinary node then estimates its position, typically, according to the intersection of various circles centered at each reference node with radii correspond to the range measurements. In general, to localize a 
node in $d$-dimensional space, the number of independent range measurements required should be at least $d+1$.

\section{- Step 3: Calibration}

The location estimate is refined e.g., using measurements from various iterations, measurement error models, mobility models, etc.

\subsection{Challenges}

Although range-based localization has been widely studied for terrestrial wireless sensor networks, existing techniques cannot be directly applied to UWSNs because of the following characteristics:

\subsubsection{Underwater environment}

While node deployment in terrestrial networks is relatively straightforward, the corresponding deployment in underwater environment encounters the following challenges:

\section{- Reference deployment in deep sea}

To localize underwater nodes deployed in the 3D sea environment, terrestrial localization techniques would require a reference node to be deployed underwater, in addition to references attached to surface buoys. This is challenging, particularly in deep sea applications, where reference nodes may need to be deployed on the sea floor at 3-4 km depth. Moreover, as replacement of batteries for submerged modems is difficult, short-range, low-power communication to achieve reasonable data transmission rates is preferred, which may limit the localization coverage. 


\section{- Node mobility}

While it is reasonable to assume that nodes in terrestrial networks remain static, underwater nodes will inevitably drift due to underwater currents, winds, shipping activity etc. In fact, nodes may drift differently as oceanic current is spatially dependent. While reference nodes attached to surface buoys can be precisely located through GPS updates, it is difficult to maintain submerged underwater nodes at precise locations. This may affect localization accuracy, as some distance measurements may have become obsolete by the time the node position is estimated.

\section{- Inter-node time synchronization}

Since GPS signals are severely attenuated underwater, it cannot be used to time-synchronize nodes deployed underwater to compensate for clock drifts due to both offset and skew. Consequently, the accuracy of ToA-based range measurement may be affected.

\section{- Signal reflection due to obstacles and reflective surfaces}

In near-shore or harbor environments, where obstacles may exist between nodes, non-line-of-sight (NLOS) signals reflected from reflecting object (e.g., sea surface, harbor wall) can be mistaken for LOS signals, and may significantly impact the accuracy of range measurement.

\subsubsection{Underwater acoustic propagation}

Unlike RF propagation in terrestrial networks, underwater acoustic propagation possesses the following unique characteristics (Etter, 2003): 


\section{- Long propagation delay}

Since the speed of sound underwater is five orders of magnitude lower than RF propagation over the air, measurement errors due to node mobility may become significant.

\section{- Multipath fading and shadowing}

The underwater acoustic channel is a frequency selective channel with a delay spread of the order of hundreds of taps ${ }^{2}$. Multipath models as well as actual measurements taken from sea trials show that the energy of the direct path of the channel's impulse response is not always the strongest (e.g., see Figure 3). As a result, multipath (indirect) signals can be mistaken for the direct signal and may significantly impact the accuracy of distance estimation.

\section{- Sound speed variation}

Unlike the speed of light which is constant, the speed of sound underwater varies with water temperature, pressure and salinity, giving rise to refraction. Without measuring the sound speed, the accuracy of distance measurements based on time-of-arrival approaches may be degraded.

\section{- Highly unreliable and asymmetric Signal-to-Noise Ratio}

For a node pair $(i, j)$, the signal attenuation in underwater acoustic

\footnotetext{
${ }^{2} \mathrm{~A}$ tap refers to the extraction of the signal at a certain position within the channel's impulse response delay-line.
} 
channels is widely modeled as (Burdic (2002)):

$$
\mathrm{TL}\left(d_{i, j}\right)=10 \lambda \log _{10}\left(\frac{d_{i, j}}{1 \mathrm{~m}}\right)+\alpha d_{i, j} \quad \mathrm{~dB},
$$

where TL is the transmission loss, $d_{i, j}$ is the distance from node $i$ to node $j, \lambda$ is the propagation loss parameter and $\alpha$ is the absorption loss parameter. The parameter, $\lambda$, depends on the structure of the underwater medium, such that in shallow water or when transmitting from one sound speed layer to another (Burdic (2002)), it can exceed 2, which is a typical value for omnidirectional propagation in free-space. The parameter $\alpha$ increases with the carrier frequency and is affected by the salinity as well as water temperature, among other factors. Since the ambient noise level is depth dependent and decreases with frequency and can be modeled as $50-\log (f)[\mathrm{dB}]$, where $f$ is the carrier frequency (Stojanovic (2006)), small variations in $f$ can affect the SNR signifi-

cantly. As a result, bandwidth is very limited in underwater acoustic channels and communications links are unreliable. Moreover, nodes $i$ and $j$ may be at different depths, the respective noise levels may be different, giving rise to asymmetric SNR. Typical values of SNR as a function of the transmission distance and the carrier frequency are shown in Figure 4.

\section{- Asymmetric power consumption}

Unlike RF modems, acoustic modems typically consume much more power (order of tens of watts) in transmit mode compared to receive mode (order of milliwatts). This asymmetry in power consumption makes it preferable for ordinary nodes to be localized through passive 
/ silent listening.

\section{- Low bit rate}

Compared to RF communications, the bit rates achievable with acoustic communications is significantly lower. As a result, the communications overhead is much higher and becomes more significant in underwater acoustic communications.

Figure 5 maps the above challenges to each desirable localization performance metric.

In the following, we further categorize range-based underwater localization schemes as (i) static vs mobile references and (ii) single vs multi-stage schemes as follows:

\subsection{Single-Stage, Static References}

Schemes that fall under this category rely on reference nodes deployed on surface buoys whose locations are determined via GPS. In "single-stage" underwater localization, all ordinary nodes are localized via message exchanges directly with the reference nodes. Once they are localized, they remain passive and do not contribute towards localizing other ordinary nodes. The key innovations of proposed schemes within this category lie in how they address localization inaccuracy due to measurement errors and transmission losses as well as the need for time synchronization.

\subsubsection{UPS-based Underwater Localization}

The "Underwater Positioning System" (UPS) (Cheng et al., 2008) is one such scheme that can be used for localization as well as for navigation in 
UWSNs. It provides "silent positioning", i.e., ordinary nodes do not transmit any beacon signal and just listen to the broadcasts of reference nodes to self-position, reducing the communication costs. Moreover, by applying TDoA over multiple beacon intervals, UPS does not require any time synchronization amongst nodes. The effects of NLOS due to multipath fading are mitigated by considering an Ultra Wideband Saleh-Valenzuela (UWBSV) model (Saleh and Valenzuela (1987)) for underwater acoustic fading channel. Assuming that the effects of node mobility and receiver system delay on range measurements are negligible, the scheme has been shown to exhibit low positioning error by executing over multiple iterations.

However, even though only four reference nodes are required to localize a 3D UWSN, at least one has to be on the seabed, which can be infeasible for deep water. Moreover, the assumption that four reference nodes must provide communication coverage over the entire network limits the area of interest and renders this scheme unscalable to large-scale UWSNs. In addition, the scheme relies on "reactive beaconing", i.e., reference nodes beacon in response to receiving other reference nodes' beacons, making it susceptible to failure due to transmission losses that are prevalent in harsh underwater acoustic channels. The reactive beaconing mechanism and the corresponding timing diagram of UPS are illustrated in Figure 6 for 2D localization.

In Tan et al. (2009), the authors identified the limitations of UPS in harsh and dynamic underwater acoustic channels and proposed enhancements - termed (E-UPS) - that improve the robustness of localization while preserving the "silent" property. This is achieved by (i) introducing redundancy through deploying more reference nodes; (ii) devising a dynamic 
mechanism for leader reference node identification; and (iii) introducing a time-out mechanism to trigger beaconing in the event of transmission loss. Simulation results show that, under typical channel conditions experienced underwater, E-UPS performs better than UPS in all aspects of localization performance.

The authors in Cheng et al. (2008) also investigated the uniqueness of positioning with UPS via extensive simulations and found that there exist regions where the position of nodes cannot be uniquely determined. In Tan et al. (2010), the authors investigated this "uniqueness" issue formally, and proposed a "Wide coverage Positioning System" (WPS) that increases the unique localization space by using a $5^{\text {th }}$ reference node, but trades off in terms of localization speed and communication costs when compared with UPS.

\subsubsection{Model-based Localization}

Instead of using commonly-adopted circle-based (see Figure 7(a)), leastsquares algorithm for location estimation and calibration, the authors in Bian et al. (2009, 2010) proposed (i) a hyperbola-based approach (HYP) (see Figure 7(b)) in Step 2 and (ii) applying known probabilistic models for measurement errors in Step 3 (PBL) to improve localization accuracy. The premise is that when range measurement errors due to imperfect time synchronization, or varying speed in acoustic transmission exist, two hyperbolas always intersect with each other with one cross point, or partial solution, while two circles will likely intersect with either two or zero cross point(s). By modeling measurement errors due to imperfect time synchronization using a normal distribution, it is shown that it is easier to find hyperbola partial 
solutions compared to circle-based partial solutions to improve the location estimation.

\subsection{Multi-Stage, Static References}

Unlike single-stage schemes, ordinary nodes do not need to communicate directly with reference nodes for multi-stage localization. Once ordinary nodes are localized, they may become "new" reference nodes and help to localize other ordinary nodes - hence, nodes are active. Unlike the singlestage schemes, the key innovations of proposed schemes within this category lie in (i) how ordinary nodes qualify as new reference nodes and (ii) which new reference nodes are used for localization so as to trade-off between minimizing error propagation and delay while maximizing coverage and energy efficiency. In addition, several schemes also addressed the issue of reference node deployment.

In the "GPS-less localization protocol" (GPS-less) (Othman, 2008), the author proposed a two-phase protocol based on a single reference node as follows: (i) Start a discovery process from the initial reference node and build a relative coordinate system using the first three discovered ordinary nodes; (ii) Extend the node discovery by selecting ordinary nodes according to their proximity from the new reference nodes. Once the coordinate system is determined, each ordinary node requires at least two known distance measures from reference nodes to compute its location. The drawbacks of this technique are that ordinary nodes only know their relative coordinates from the primary seed node and the first-stage discovery protocol requires high volume of message exchange. Moreover, the nodes have to be static, and any node mobility could degrade the performance drastically, especially 
in terms of accuracy.

In Mirza and Schurgers (2008), the authors proposed a Motion-Aware Sensor Localization (MASL) scheme that specifically accounts for position error due to non-concurrent distance measurements, which can occur due to node mobility. In fact, ranging experiments with a pair of WHOI micromodems (Freitag et al., 2005) at Mission Bay, San Diego, indicated that a zero-mean Gaussian model is well suited for ranging errors, and this forms the premise to the proposed scheme. However, unlike most proposed schemes, MASL is targeted at offline applications, as it is computationally intensive.

In Bian et al. (2007), the authors proposed a joint localization and synchronization scheme (L-S) for 3D UWSNs. The 3D network is partitioned into cells, and localization is performed at the cell level. Each ordinary node qualifies as a new reference and achieves time synchronization as soon as it receives beacons from five reference nodes. The authors determined the required sensor node density, as well as cell partitioning in order to localize all nodes. While not implemented in this study, the authors identified the need to reduce error propagation and suggested various methods such as new reference node selection and weighted least squares approach, e.g., by weighting reference node's contribution to localization according to its tier number (proximity from the ordinary node).

\subsubsection{Localization for Hierarchical UWSNs}

In Zhou et al. (2010), the authors consider a hierarchical architecture for a large-scale UWSN comprising reference nodes attached to surface buoys, submerged anchor nodes (assumed to be within communication range and can be localized accurately by the reference nodes using any single-stage scheme 
described in Section 2.2) and ordinary nodes. They proposed a distributed large-scale localization scheme (LSL) to localize ordinary nodes with the help of localized anchor (reference) nodes.

To alleviate the effects of error propagation, when an ordinary node obtains its estimated position from a set of reference nodes, it computes a confidence value, $\eta$, where $0<\eta \leq 1$, by normalizing the position error with the sum of the Euclidean distance between itself and the reference nodes (see Eqn (2)). This value reflects the confidence in position estimation (with a higher value indicating higher confidence), and permits the node to qualify as a new reference node provided $\eta \geq \eta_{0}$, where the choice of the threshold $\eta_{0}$ represents the tradeoff amongst localization coverage, accuracy and communication costs. However, we note that the confidence value (fixed at 1 for anchor nodes) is a subjective measure since the ordinary node estimates both $l_{i}$ as well as its location.

Referring to the notations in Figure 6, each reference node periodically broadcasts a beacon that comprises its ID, position as well as its confidence value. Each ordinary node $S$ estimates its distance, $l_{i}$, from a reference node $R_{i}$ (i) based on ToA approach upon receiving its beacon or (ii) using 3D Euclidean distance estimation if they are more than one-hop apart. The latter process is included to improve the likelihood of an ordinary node receiving at least four distance estimates (for 3D localization) from reference nodes to estimate its position, particularly in anisotropic networks. When this happens, node $S$ selects those with the highest confidence value to estimate its position, $\left(\tilde{x}_{S}, \tilde{y}_{S}, \tilde{z}_{S}\right)$ and computes the position error, $\delta$ as follows:

$$
\delta=\sum_{i}\left|\left(\tilde{x}_{S}-x_{i}\right)^{2}+\left(\tilde{y}_{S}-y_{i}\right)^{2}+\left(\tilde{z}_{S}-z_{i}\right)^{2}-l_{i}^{2}\right|
$$


Its confidence value, $\eta$, is then computed as follows:

$$
\eta= \begin{cases}1, & \text { node is anchor } \\ 1-\frac{\delta}{\sum_{i}\left(\tilde{x}_{S}-x_{i}\right)^{2}+\left(\tilde{y}_{S}-y_{i}\right)^{2}+\left(\tilde{z}_{S}-z_{i}\right)^{2}}, & \text { otherwise. }\end{cases}
$$

If $\eta \geq \eta_{0}$, node $S$ qualifies as a new reference node, and begins its periodic beaconing, subject to a maximum of $N$ messages to limit the localization overhead.

Through extensive simulations, the authors illustrated the tradeoffs between localization coverage, accuracy and communication costs with the choice of the threshold value, $\eta_{0}$ : For an UWSN with $5 \%$ anchor nodes, node density of 13 , and $N=5$, a value of $\eta_{0}=0.8$ achieves high coverage of $80 \%$ with low communication costs at the expense of high localization error of $0.45 R_{c}$ (where $R_{c}$ is the communication range), while $\eta_{0}=0.96$ yields lower localization error of $0.35 R_{c}$ at the expense of lower coverage of $55 \%$ and higher communication costs. Hence, the choice of $\eta$ depends on whether high coverage is needed (e.g., where location information is used in routing) or high accuracy is needed (e.g., for association with sensed data).

The authors also showed that the convergence property of the proposed scheme is tightly linked with the message limit, $N$; in fact, there exists a critical $N_{0}$, for which beyond this value, the communication costs continue to increase significantly without any corresponding improvement in localization coverage. From the simulations, $N_{0}=5$ for an UWSN with $10 \%$ anchor nodes, node density of 13 and $\eta$ ranging from 0.7 to 0.99 .

The "Scalable Localization scheme with Mobility Prediction" (SLMP) (Zhou et al., 2008) introduces mobility prediction based on predictable mobility patterns of underwater objects in sea shore environments (Novikov and 
Bagtzoglou, 2006) to the LSL scheme. Basically, if the mobility pattern of nodes is precise enough, i.e., they follow the mobility pattern assumed, then they do not broadcast updates unnecessarily, reducing communication costs. While the confidence value remains as the criteria for qualification and selection as a reference node, the authors proposed an additional criteria for reference node selection based on arrival time-stamp: if the arrival time of the last localization message is too far from the current time, the reference node will be deleted from the list used for localization.

The timing diagram for both LSL and SLMP are shown in Figure 8(a).

\subsubsection{Projection-based Localization}

While the above schemes have addressed the coverage limitations of singlestage schemes, they rely on the deployment of reference nodes in the sea, which is challenging. This need is delineated by the following projectionbased schemes that only require the deployment of reference nodes attached to three surface buoys and pressure sensors attached to ordinary nodes to measure their depth.

In Cheng et al. (2009a), the authors propose a distributed localization technique termed "Underwater Sparse Positioning" (USP) that employs a projection method, which transforms the 3D underwater positioning problem into its 2D counterpart. The initial reference nodes bootstrap the localization procedure by broadcasting their positions once deployed. Upon receiv-

ing the broadcast from $R_{i}$, ordinary node $S$ projects it onto $R_{i}^{\prime}$ on its own horizontal plane, as illustrated in Figure 9(a). As long as the projection is non-degenerative, which is likely in sparse UWSNs, simple bilateration can be used to localize ordinary nodes. Each localized ordinary node then be- 
comes a new reference node, and all reference nodes are used in the projection mechanism for localization. The procedure runs for $M$ iterations: the time interval in each iteration is divided into three parts as shown in Figure 9(b).

In Cheng et al. (2009b), the authors combine USP and UPS to give rise to a multi-stage silent positioning system LSLS that (i) does not require time synchronization, (ii) only requires three surface buoys, (iii) incorporates smart anchor selection, and (iv) considers sound speed variation with temperature, salinity and depth using the model of Coppens (Coppens, 1981).

A similar projection mechanism is used in the 3D localization algorithm 3DUL proposed in Isik and Akan (2009). However, unlike USP that (i) employs a predetermined number of iterations and periodic broadcasting of the three surface buoys to the whole network, and (ii) assumes inter-node time synchronization, 3DUL (i) uses two-way messaging for ranging (and therefore does not require time synchronization), (ii) estimates the sound speed through the use of (Conductivity, Temperature and Depth) sensors, and (iii) limits the duration with which a new reference node remains as reference according to its movement characteristics. As long as the projected reference locations fall on a robust virtual anchors plane, the ordinary node will be localized and become a new reference node. Referring to Figure 9(a), quadrilateral $S R_{1}^{\prime} R_{2}^{\prime} R_{3}^{\prime}$ forms a robust virtual plane if all four sub-triangles $\Delta R_{1}^{\prime} R_{2}^{\prime} R_{3}^{\prime}, \Delta S R_{1}^{\prime} R_{2}^{\prime}, \Delta S R_{1}^{\prime} R_{3}^{\prime}$ and $\Delta S R_{2}^{\prime} R_{3}^{\prime}$ are robust, i.e., they satisfy the following condition:

$$
a \sin ^{2} \theta>d_{\min }
$$

where $a$ is the length of the shortest side, $\theta$ is its angle with the hypotenuse and $d_{\min }$ is a threshold that depends on measurement noise (Moore et al., 
2004). For example, for $\Delta S R_{2}^{\prime} R_{3}^{\prime}$, the shortest side is $S R_{3}^{\prime}$, and $\theta$ is the angle between $S R_{3}^{\prime}$ and $S R_{3}$.

The two-way message exchange between an ordinary node and a reference node is shown in Figure 8(b). "wakeup" messages are sent by the reference node to declare its presence. Each ordinary node that receives this message will respond with a "request" message and note the time instance it was sent. The reference node responds with a "response" message that includes its coordinates. Upon receiving several "response" messages, the ordinary node can then estimate its position without the need for inter-node time synchronization as long as the round trip time and the speed of sound can be accurately estimated.

Unlike the L-S and GPS-less schemes, USP and 3DUL do not employ any smart anchor selection scheme, as it would increase the complexity while degrading the performance in dynamic underwater acoustic channel environment. However, in LSLS, new reference nodes for reactive beaconing are selected to minimize the overlap with the communication coverage of existing reference nodes so as to maximize localization coverage.

\subsection{Mobile References}

Although some of the above schemes no longer rely on the deployment of seabed reference nodes, the deployment of fixed reference nodes such as surface buoys is time consuming, limits the localization coverage and may be infeasible or undesirable (e.g., in tactical surveillance applications). Some of these drawbacks may be overcome by employing mobile reference(s) such as Autonomous Underwater Vehicles (AUVs) (Erol et al., 2007a; Luo et al., 2008, 2010) or Dive-aNd-Rise-enabled (DNR-enabled) devices (Erol et al., 
2007b; Chen et al., 2009).

\subsubsection{AUV-assisted localization}

In the AUV-Aided localization technique proposed in Erol et al. (2007a), the sensor nodes can be dropped into the ocean and will move with the water currents while an AUV will traverse the UWSN periodically. The AUV obtains position updates by rising to the surface to use GPS, and then dives to a predefined depth and periodically performs a two-way message exchange with ordinary nodes as in 3DUL (see Figure 8(b) and Figure 10). Assuming that each ordinary node is equipped with a pressure sensor (to measure its depth), it can be localized as soon as successful two-way message exchanges take place in at least three non-collinear AUV locations. The AUV is assumed to move at a constant and known speed and is capable of estimating its position underwater accurately (within $1 \mathrm{~m}$ ) by using integrated GPS, compass and dead reckoning. Another major assumption is that each ordinary node is either static or can estimate its motion underwater.

\subsubsection{DNR-enabled localization}

Instead of AUVs, the DNR localization (DNRL) scheme (Erol et al., 2007b) uses a weight/bladder mechanism to control the diving/rising of each mobile beacon, which comprises a GPS receiver and an acoustic transceiver. These beacons update their positions at the surface, and broadcast them when they dive to a certain depth. The DNRL scheme is evaluated using a meandering current mobility model in Caruso et al. (2008), which is suitable for a large coastal environment.

In Erol et al. (2008), the authors present proxy localization (PL), which 
enhances the DNRL scheme through multi-stage localization. To minimize error propagation, localized ordinary nodes can qualify as new reference nodes only if they are below the maximum depth of the DNR beacons. Each ordinary node will then select the most recently qualified set of reference nodes with the minimum hop count from the initial reference nodes.

A single-stage scheme is proposed in Chen et al. (2009), LSL-DET, that uses the network architecture of LSL but extends the reach of surface buoys by attaching "Detachable Elevator Transceivers" (DET) to them. The concept is similar to DNRL, except that DETs that dive and rise do not contain GPS receivers, thus reducing the cost.

Although the principles of AUV-Aided and DNR-enabled schemes are similar, the AUV-Aided scheme is more flexible as it can traverse both horizontal and vertical preprogrammed routes while the mobile references in DNR-enabled schemes can only traverse vertically.

\subsection{Comparison of range-based schemes}

In this section, we compare range-based underwater localization schemes in terms of (i) how and the extent to which they address various challenges and (ii) their performance as obtained via simulation studies.

\subsubsection{Challenges addressed}

Table 1 summarizes the extent to which range-based underwater localization schemes have addressed the challenges of underwater localization outlined in Section 2.1

We observe that in most schemes, each ordinary node either employs (i) silent listening to estimate its location, assuming inter-node time synchro- 
nization or (ii) two-way messaging, without the need for time synchronization, but at the expense of higher communication overhead. However, UPSbased schemes are able to use silent listening without assuming inter-node time synchronization using reactive beaconing with TDoA measurements.

Measurement errors due to sound speed variation, NLOS signals, time non-synchronization and node mobility are handled in various ways: (i) $\mathbf{H Y P}$ and PBL assume that errors due to time non-synchronization follow a normal distribution; (ii) USP and LSLS assume normally distributed ranging errors; (iii) 3DUL, LSL, SLMP, MASL, DNRL and PL consider various node mobility models; and (iv) UPS is the only scheme that specifically considers the effects of NLOS signals by modeling the underwater acoustic channel using a UWB-SV model. Although the model assumes that the direct path signal has the strongest signal, this is not always the case in reality, e.g., see Figure 3.

Last but not least, while UPS requires a reference node to be deployed on the seabed and LSL-based schemes assume a costly deployment of submerged anchor nodes in addition to surface buoys, projection-based techniques delineate the need for seabed reference nodes, while AUV-based localization schemes completely eliminate the need for costly infrastructure.

Table 2 summarizes the mechanism used by multi-stage schemes to tradeoff between minimizing error propagation and delay while maximizing coverage and energy efficiency. The techniques are centered around (i) minimizing overlap with existing reference nodes to maximize coverage; and (ii) maximizing proximity with existing reference nodes and latest updates and minimizing positioning error to minimize error propagation. 


\subsubsection{Performance Comparison}

The various schemes are evaluated via simulations based on one or more of the following metrics:

\section{- Communication Costs}

This metric quantifies the energy efficiency of the localization scheme. We define communication costs in terms of the average number of messages transmitted per node to achieve a single localization estimation.

\section{- Coverage}

We define the coverage as the proportion of ordinary nodes that are successfully localized. In multi-stage schemes, this refers to those that qualify as new reference nodes.

\section{- Time}

This metric quantifies the time taken (either in iterations or seconds) to achieve the stated coverage.

\section{- Accuracy}

This metric quantifies the localization error, i.e., the Euclidean distance between an ordinary node's estimated and actual locations. Here, we normalize this error to the communication range.

Since the simulation scenario used in evaluating the various schemes are different, it is difficult to conduct a fair comparison amongst them. We draw on the simulation study to compare DNRL, LSL and PL in Erol et al. (2010), and present the performance comparison of selected schemes with the respective description of the simulation scenario in Table 3. 


\section{Range-free Underwater Localization Schemes}

As described in the previous section, the accuracy of range-based localization depends on the accuracy of range measurement, which could suffer from large errors due to node mobility as well as harsh underwater acoustic propagation environment. Hence, range-free schemes have been proposed that do not rely on range measurement for localization.

\subsection{Schemes based on Area Localization}

In Chandrasekhar and Seah (2006), the authors proposed a 2D Area Localization Scheme, ALS to estimate a node's position within a certain area rather than its exact location. The propagation of acoustic signals underwater is subject to losses due to spreading, absorption, dispersion, multi-path fading and Doppler effects.

Assuming a spherical attenuation model, and neglecting losses due to multi-path fading and Doppler effects, when each reference node transmits at power $P_{i}$, an ordinary node can receive the transmission as long as it falls within a circular region centered at the reference node whose radius $r\left(P_{i}\right)$ depends on the transmission power. Hence, by deploying several reference nodes that transmit beacons at multiple power levels, the plane is divided into many small sub-regions defined by intersecting circles. Each ordinary node listens and reports the ID and minimum transmit power at which it received the respective node's beacon to a central sink, which can then estimate its location. This is illustrated in Figure 11. The main limitations of this scheme are (i) it is a centralized scheme; (ii) its coverage is limited by the communication range of the reference nodes; (iii) it is model-based; 
(iv) it only provides coarse localization; and (v) it does not consider node mobility.

In Zhou et al. (2009), the authors proposed a scheme, 3-D Multi-stage Area Localization Scheme (3D-MALS) that combines the concepts of ALS and LSL-DET. It considers a hierarchical network architecture that comprises surface buoys with "Detachable Elevator Transceivers" (DET), ordinary nodes and sink nodes, and extends ALS to 3D. Simulation results demonstrate its performance gain over ALS in terms of localization accuracy. However, as with ALS, it is a model-based, centralized scheme that provides coarse localization and does not consider node mobility.

\subsection{Schemes based on directional beaconing}

While the above schemes rely on static references, the authors in Luo et al. $(2008,2010)$ proposed a 2D and 3D underwater localization scheme (UDB and LDB respectively) using a single AUV with directional beacons. Here, the AUV traverses a preprogrammed route and performs directional (vertical) beaconing periodically. The scheme assumes that the AUV moves with constant and known speed and knows its position underwater accurately using integrated GPS and INS, and that the vertical channel used in UDB is characterized by little or no time dispersion.

Figure 12 gives an illustration of how ordinary node $S$, equipped with a pressure sensor, localizes itself with LDB.

Assuming a beamwidth of $\alpha$, the radius of the circle formed by the intersection of the beam with the horizontal plane for which $S$ resides, $r_{2}$, is given as:

$$
r_{2}=\tan \left(\frac{\alpha}{2}\right) \times h_{2}
$$


where $h_{2}$ is the difference in depth between the AUV and node $S$.

We assume that the AUV traverses a straight line path and broadcasts its own location periodically, at times $t_{0}, t_{1}, \cdots, t_{7}, \cdots$. At instant $t_{i}$, node $S$ would record the AUV's coordinates, $\left(x_{i}, y_{i}\right)$, if it can hear them, i.e., if it lies within the circle of radius $r_{2}$ centred at $\left(x_{i}, y_{i}\right)$. According to Figure 12, node $S$ first hears the AUV's beacons when it transmits at $\left(x_{1}, y_{1}\right)$ at time $t_{1}$ and last hears them when it transmits at $\left(x_{5}, y_{5}\right)$ at time $t_{5}$. Accordingly, it estimates its position, $\left(\tilde{x}_{S}, \tilde{y}_{S}\right)$, as follows:

$$
\begin{aligned}
& \tilde{x}_{S}=\frac{x_{1}+x_{5}}{2} \\
& \tilde{y}_{S}=y_{1}+\frac{\sqrt{r_{2}^{2}-\frac{x_{5}-x_{1}+2 d^{2}}{2}}+\sqrt{r_{2}^{2}-{\frac{x_{5}-x_{1}}{2}}^{2}}}{2},
\end{aligned}
$$

where $d$ is the distance traversed by the AUV between successive beaconing instances.

\subsection{Finger-printing based schemes}

A different variant of range-free localization schemes based on finger-

printing (PM, PCA, PF) have recently been proposed in Lee et al. (2009b,a,c). Such schemes involve an offline (or training) stage prior to the online (or prediction) stage. The setup comprises an acoustic signal source capable of transmitting at $M$ different frequencies, and $L$ reference locations with known positions and a node (receiver) to be localized.

During the offline stage, the receiver is placed at each reference location (with known position), collects $N$ samples of acoustic communication signals at each frequency to constitute an $M \times N$ acoustic-signal map. All the signals are projected onto the eigenspace for Principal Component Analysis, where 
$M^{\prime}$ signals corresponding to the largest eigen values are extracted in order to reduce the complexity and noise effects. This is repeated at the $L$ reference locations.

In the online stage, the receiver is placed at an unknown location (within the reference location space) and collects acoustic communication signals from $M$ different frequencies to establish a signal vector, from which $M^{\prime}$ principal components are extracted as in the offline stage. A likelihood function is used to express the probability that the unknown location corresponds to a reference one, and the unknown location can then be estimated by the "probabilistic-weighted" summation of different reference locations.

The efficacy of the proposed scheme is verified in actual experiments in a water tank. However, the practical use of this scheme is limited since the actual underwater acoustic channel in the sea is highly time varying (Stojanovic, 2003).

\section{Commercial Underwater Positioning System}

Commercially available underwater acoustic positioning systems have existed as early as in the 1960s (Vickery, 1998) and are commonly used in various applications, including oil and gas exploration, salvage operations, marine sciences and archaeology, security and military operations. These systems are used for tracking and navigation of divers and/or underwater vehicles. These systems measure the distances and/or directions of the diver or vehicle being tracked from a set of transponders (reference points), referred to as baseline stations, and then trilateration is done to determine its position. Based on the distance between the baseline stations, these systems 
are broadly categorized into: Long Baseline (LBL), Short Baseline (SBL) and Ultra Short Baseline (USBL). In the following discussion, we use the term "object" to refer to the item (i.e., diver or vehicle) being tracked.

\subsection{Long Baseline Systems}

The name derives from the fact that the distance between the baseline stations is long or similar to the distance between object and transponders. Baseline distance typically ranges from 50m to more than 2,000m (Rowan, 2008). The baseline transponders are typically deployed on the seafloor along the edge of the area of operation. An interrogating signal from the object (transponder) is sent. Upon receiving it, the transponder (object) sends a reply. The signal propagation times are then used to compute the distances between the object and transponders. Together with depth data from pressure gauges, the position of the object can then be computed using trilateration. This position is relative to the baseline transponders but can be easily converted into geo-referenced coordinates if the geographic positions of the baseline transponders are known a priori. The baseline transponders can also be mounted in fixed relative positions on a moving platform like a ship for applications like In-Water Ship Hull Inspections and other specialized tasks. LBL systems are independent of water depth and provide high accuracy of better than $1 \mathrm{~m}$, with the capability of achieving accuracies of a few centimetres. This exceeds the accuracies of USBL and SBL systems. Manufacturers of LBL systems include Applied Acoustics (Transponders for LBL systems), Desert Star Systems LLC (AquaMap LBL systems), LinkQuest Inc. (Pin-

point LBL System), Nautronix (NASNet LBL system), Sonardyne (Fusion LBL system) and Sonatech (seafloor transponders). A survey of LBL systems 
can be found in (Hydro, 2008a).

The GPS Intelligent Buoys (GIB) can be viewed as inverted LBL devices. Instead of deploying the baseline transponders on the seafloor, they are installed on GPS equipped sonobuoys that are either drifting or moored (Thomas, 1998; Alcocer et al., 2006). In a typical deployment scenario, several GIBs are deployed over a given area of operation where the total number required is determined by the size of the operation area and the desired accuracy (accuracies of centimetre to meter level in realtime is achievable.) The position of the tracked object is calculated in realtime based on the acoustic signals transmitted by the underwater object. GIB uses one-way acoustic signal transmission from object (emitter) to buoys as compared to the round-trip transmission of LBL, SBL and USBL, making it less susceptible to reflections from surface or other undersea structures. The GIB system is patented technology that has been developed by ORCA Instrumentation and French R\&D firm Advanced Concept and System Architecture (ASCA); ACSA Underwater GPS (subsidiary of the ALCEN group) manufactures it.

\subsection{Short Baseline Systems}

SBL systems are characterized by inter-baseline transponder distance of $20 \mathrm{~m}$ to $50 \mathrm{~m}$. These systems are mounted on floating platforms like boats, ships or barges, and are used for tracking underwater targets. Like LBL systems, they use round-trip signal propagation time of acoustic signals between transponders and target to compute the distance and then trilateration to determine the position, often with supplementary depth data from a pressure sensor. The accuracy of SBL system improves as the distance between baseline transponders increases and can achieve similar performance levels 
as seafloor mounted LBL systems. Conversely, when used on smaller vessels that reduce the inter-baseline transponder distance, the accuracy also deteriorates. SBL systems are popular with research groups and have been used in Antartica expeditions to track vehicles operating under the ice, e.g. Project SCINI (scini.mlml.calstate.edu).

\subsection{Ultra Short Baseline Systems}

USBL is by far the most popular category of underwater positioning systems due to its ease of use. A USBL system comprises two elements, a transceiver with an array of transducers that are typically less than $10 \mathrm{~cm}$ apart, thus giving the ultra short baseline, and a transponder/responder. The transceiver sends an acoustic pulse and when the transponder (mounted on the tracked object) detects this pulse, it replies with its own acoustic

pulse. The round-trip propagation time of the initial acoustic pulse and the reply is used to compute the distance between them. To measure the direction of the transponder from the transceiver, the transceiver uses a method called phase-differencing within this transducer array to calculate the angle to the underwater transponder. The position of the undersea object (transponder) is then obtained using the range and angle from the transceiver to the transponder. What the system gains in ease of use and deployment, it loses in the level of positioning accuracy. Manufacturers of USBL systems include Nautronix, Sonardyne, IXSEA (GAPS pre-calibrated Ultra-Short BaseLine), Applied Acoustics (EASYTRAK USBL), LinkQuest (TrackLink USBL), Tritech (Micron Nav), Kongsberg (HiPAP - High Precision Acoustic Positioning), and EvoLogics (USBL Acoustic Modem). A survey of USBL systems can be found in (Hydro, 2008b). 


\section{Summary}

In this paper, we conducted a survey of recently proposed localization schemes specifically designed for UWSNs. We identified several of the challenges that need to be overcome for underwater localization schemes to be fast and accurate, have low communication costs, provide wide coverage and be feasible. In addition to classifying the schemes under (i) range-based, (ii) range-free and (iii) finger-printing based schemes, we also further classify range-based schemes as (i) single vs multi-stage and (ii) static vs mobile references.

Although all the proposed schemes demonstrate good performance in simulations, they have not been evaluated under the same conditions, nor with the same initial assumptions. For example, some schemes require many initial reference nodes to achieve good performance, which could be too costly for the UWSN application. On the other hand, other schemes assume that the entire network can be covered by only a few reference nodes, which limits the deployment area of the UWSN and may incur large communication costs. Hence, the localization scheme should be chosen to tailor to the needs of the UWSN application.

In general, schemes that rely solely on static references achieve better localization accuracy at the expense of higher deployment costs. These schemes are suitable (i) for early warning systems against disasters such as tsunami or seaquakes, or (ii) to assist underwater navigation (locate dangerous rock or shoals) especially if reference nodes are on the seabed. Schemes that rely on mobile references can be deployed quickly and are thus suited for emergency applications although the water currents will have more negative impacts on 
their performance than for the former schemes. These schemes can be easily used to sample some underwater areas or for distributed tactical surveillance where sensors can monitor some specific underwater areas to detect intrusion, target or reconnaissance. Besides, if an AUV is deployed among the sensors, it can be used for more specific missions such as underwater ordinance reconnaissance, rapid environmental assessment and detection of potential threats.

To make our survey more complete, we have also included a discussion of commercially available underwater positioning systems, which can be classified under long baseline, short baseline and ultra short baseline systems. We described the mechanism of each type of system, and outlined the performance tradeoffs.

\section{Open Problems and Research Challenges}

While challenges associated with reference node deployment, time synchronization, and asymmetric power consumption in acoustic modems have been addressed to some extent in the proposed schemes, in our view, the following challenges should be, but have not been, fully addressed:

\section{- Sound Speed Variation}

While most range-based localization techniques assume a constant speed of sound underwater, it actually depends on the temperature, pressure and salinity. The authors in Isik and Akan (2009) and Mackenzie (1981) investigated the impact of sound speed variation on the localization accuracy using the nine-term equation in Mackenzie (1981) and the 
Coppen's model (Coppens, 1981) respectively. For a fair performance comparison of all schemes, they should be evaluated using a common and accurate sound speed model.

\section{- Inter-node Time Synchronization}

Localization schemes that rely on silent positioning to minimize communication overhead assume that nodes are time-synchronized. However, unlike surface nodes that can be time-synchronized via GPS updates, submerged nodes cannot be time-synchronized, and their clocks are subject to skew as well as offset. Although time synchronization protocols (e.g., in Syed and Heidemann (2006); Chirdchoo et al. (2008b)) have been proposed for underwater acoustic networks, they should be incorporated into localization schemes.

\section{- Node mobility model}

Node mobility due to water currents, which presents one of the greatest challenges for underwater localization, has only been accounted for up to various degrees. Although most schemes assume static nodes, the LSL scheme assumes a simple (and unrealistic) mobility model, the SLMP scheme considers mobility patterns taken from a seashore environment (Novikov and Bagtzoglou, 2006) while the DNRL and PL schemes consider a meandering current mobility model (Caruso et al., 2008), which is suited for large coastal environment. Based on actual ranging experiments with acoustic modems in Mission Bay, San Diego, the MASL scheme assumes a zero mean Gaussian model for ranging errors resulting from node mobility. The above indicates that in differ- 
ent shore environments, node mobility exhibits different characteristics. Moreover, to the best of our knowledge, equivalent mobility models for deep sea environments do not exist.

\section{- Impact of Medium Access Control}

Another important challenge that has not been fully addressed is Medium Access Control (MAC) to resolve contention, particularly in multi-stage localization schemes for dense UWSNs. Although MAC schemes have been proposed specifically for UWSNs (Molins and Stojanovic, 2006; Chirdchoo et al., 2008a; Chen and Wang, 2007), to support multi-stage underwater localization schemes, MAC schemes also have to coordinate the node transmissions such that each ordinary node can receive the required number of beacons from reference nodes as quickly as possible while ensuring that reference nodes do not transmit beacons redundantly. However, MAC protocols will inevitably introduce delays in transmission, and affect the accuracy of localization schemes that rely on two-way messaging. Thus, the trade-offs between communication overhead, localization latency and accuracy introduced by the MAC protocol has to be carefully evaluated.

\section{- Impact of Channel Structure}

The underwater acoustic channel is a frequency selective time-varying channel. Since the localization process requires range measurement, using either ToA, TDoA or RSSI techniques, the structure of the channel may affect the accuracy of the localization process. Since range is measured based on the ToA of the direct path or its received power, 
it is essential to lock on the location of the direct path of the received signal. Existing protocols, e.g., Cheng et al. (2008, 2009a), assume that the direct path is the strongest path and thus it's location is easy to estimate. In fact, by using the UWB-SV model to characterize the underwater acoustic channel, the authors in Cheng et al. (2008) attempt to substantiate the above claim. However, multipath fading can lead to destructive interference, and as a result, the energy of the direct path of the channel's impulse response is not always the strongest. This effect was observed in real measurements taken from sea trials (e.g., Figure 3). Hence, designated mechanisms to lock on the direct path are needed.

Range measurement relies on the premise that direct (LOS) signals always exist. However, the presence of structures and obstacles in the underwater channel may result in the loss of the direct signal, in which case only NLOS signals are detected at the receiver. As a result, NLOS signals can be mistaken for LOS signals and may significantly impact the accuracy of distance estimation. In Diamant et al. (2010), a hybrid algorithm that utilizes both RSSI and ToA measurements has been proposed. The algorithm assumes that the power of the NLOS signal is much more attenuated than that of the LOS signal due to the target strength and spreading loss characteristics of the reflecting surface, and classifies communication links as NLOS and LOS links according to the relation between the ToA and RSSI measurements. The algorithm was tested in a real harbor environment and showed good performance. However, this algorithm relies on the existence of 
an attenuation model which might not be applicable to all environments and thus more research should be done in this area.

\section{- Performance Evaluation}

Last, but not least, the proposed range-based and range-free schemes have only been evaluated analytically or via numerical simulations (under different scenarios) while finger-printing based schemes have been evaluated in a water tank. For a fair evaluation of the schemes, they should be evaluated using available simulators for underwater acoustic networks (e.g., Harris and Zorzi (2007)) under a common simulation scenario. Actual implementation and experimentation of the proposed schemes in the sea is the natural next step to establish the feasibility and actual performance achievable with these schemes.

\section{References}

Akyildiz, I.F., Pompili, D., Melodia, T., 2007. State of the Art Protocol Research for Underwater Acoustic Sensor Networks (Invited Paper). ACM MC2R 11, 11-22.

Alcocer, A., Oliveira, P., Pascoal, A., 2006. Underwater Acoustic Positioning Systems Based on Buoys with GPS, in: Proceedings of the Eighth European Conference on Underwater Acoustics (ECUA), Carvoerio, Portugal.

Bian, T., Liu, W., Jin, J., Wang, Y., Mo, Y., 2007. Localization and Synchronization for 3D Underwater Acoustic Sensor Networks. LNCS , 622$631[\mathbf{L}-\mathrm{S}]$. 
Bian, T., Venkatesan, R., Li, C., 2009. Design and Evaluation of a New Localization Scheme for Underwater Acoustic Sensor Networks. Proc. of the IEEE Globecom , 5043-5047[HYP].

Bian, T., Venkatesan, R., Li, C., 2010. An Improved Localization Method Using Error Probability Distribution for Underwater Sensor Networks. Proc. of the IEEE ICC [PBL].

Burdic, W.S., 2002. Underwater Acoustic System Analysis. Peninsula Publishing.

Caruso, A., Paparella, F., Vierra, L., Erol, M., Gerla, M., 2008. The Meandering Current Mobility Model and Its Impact on Underwater Mobile Sensor Networks. Proc. of the IEEE INFOCOM , 221-225.

Chandrasekhar, V., Seah, W.K.G., 2006. Area Localization Scheme for Underwater Sensor Networks. Proc. of the IEEE OCEANS Asia Pacific Conference $[\mathbf{A L S}]$.

Chandrasekhar, V., Seah, W.K.G., Choo, Y.S., Ee, H.V., 2006. Localization in Underwater Sensor Networks - Surveys and Challenges. Proc. of the WUWNet , 33-40.

Chen, K., Zhou, Y., He, J., 2009. A Localization Scheme for Underwater Wireless Sensor Networks. Int'l Journal of Adv. Sc. and Tech. 4, 9-16. [LSL-DET].

Chen, Y.J., Wang, H.L., 2007. Ordered CSMA: A Collision-Free MAC Protocol for Underwater Acoustic Networks. Proc. of MTS/IEEE OCEANS 
Cheng, W., Teymorian, A.Y., Ma, L., Cheng, X., Lu, X., Lu, Z., 2009a. 3D Underwater Sensor Network Localization. IEEE Trans. on Mobile Computing 8, 1610-1621. [USP].

Cheng, W., Thaeler, A., Cheng, X., Liu, F., Lu, X., Lu, Z., 2009b. TimeSynchronizaton Free Localization in Large Scale Underwater Acoustic Sensor Networks. IEEE Intl. Conf. on Dist. Sys. Wkshps , 80-87[LSLS].

Cheng, X., Shu, H., Liang, Q., Du, D., 2008. Silent Positioning in Underwater Acoustic Sensor Networks. IEEE Trans. Veh. Technol. 57, 1756-1766. [UPS].

Chirdchoo, N., Soh, W.S., Chua, K.C., 2008a. MACA-MN: A MACA-based MAC Protocol for Underwater Acoustic Networks with Packet Train for Multiple Neighbors. Proc. of IEEE VTC-Spring , 46-50.

Chirdchoo, N., Soh, W.S., Chua, K.C., 2008b. MU-Sync: A Time Synchronization Protocol for Underwater Mobile Networks. Proc. of the WUWNet , $35-42$.

Coppens, A., 1981. Simple Equations for the Speed of Sound in Neptunian Waters. J. Acoust. Soc. Am. 69, 862-863.

Diamant, R., Horev, L., 2005. Emulation System for Underwater Acoustic Channel. Proc. of the Undersea Defence Technologies Europe Conference (UDT) .

Diamant, R., Tan, H.P., Lampe, L., 2010. NLOS Identification Using a Hybrid ToA-Signal Strength Algorithm for Underwater Acoustic Localization. Accepted for publication at the MTS/IEEE Oceans Seattle . 
Erol, M., Oktug, S., Vieira, L.F.M., Gerla, M., 2010. Performance Evaluation of Distributed Localization Techniques for Mobile Underwater Acoustic Sensor Networks. Ad Hoc Networks .

Erol, M., Vieira, L.F.M., Caruso, A., Paparella, F., Gerla, M., Oktug, S., 2008. Multi Stage Underwater Sensor Localization using Mobile Beacons. Proc. of the IEEE SENSORCOMM , 710-714[PL].

Erol, M., Vieira, L.F.M., Gerla, M., 2007a. AUV-Aided Localization for Underwater Sensor Networks. Proc. of the WASA , 44-51[AUV-Aided].

Erol, M., Vieira, L.F.M., Gerla, M., 2007b. Localization with Dive'N'Rise (DNR) Beacons for Underwater Acoustic Sensor Networks. Proc. of the WUWNet , 97-100[DNRL].

Etter, P.C., 2003. Underwater Acoustic Modeling and Simulation. Spon Press. 3 edition.

Freitag, L., Grund, M., Singh, I., Partan, J., Koski, P., Ball, K., Bagtzoglou, A.C., 2005. The WHOI micro-modem: An Acoustic Communications and Navigation System for Multiple Platforms. Proc. of MTS/IEEE OCEANS

Gu, H., Shi, Y., Chen, Y., Wang, B., Jiang, W., 2006. Cicada: A HighlyPrecise Easy-Embedded and Omni-Directional Indoor Location Sensing System. LNCS: Advances in Grid and Perv. Computing 3947, 385-394.

Harris, A.F., Zorzi, M., 2007. Modeling the Underwater Acoustic Channel in ns2. Proc. of NSTools 321, 128-137. 
Hightower, J., Borriello, G., 2001. A Survey and Taxonomy of Location Systems for Ubiquitous Computing. Technical Report UW-CSE 01-08-03. University of Washington, Seatle.

Hydro, 2008a. Product survey - long baseline systems. Hydro International http://www.hydro-international.com/productsurvey/id12Long_Baseline_Systems.html.

Hydro, 2008b. Product survey - ultra short baseline systems. Hydro International http://www.hydro-international.com/productsurvey/id13UltraShort_Baseline_Systems.html.

Isik, M.T., Akan, O.B., 2009. A Three Dimensional Localization Algorithm for Underwater Acoustic Sensor Networks. IEEE Trans. Wireless Comms. 8, 4457-4463. [3DUL].

Lee, K.C., Ou, J.S., Huang, M.C., 2009a. Underwater Acoustic Localization by Principal Components Analyses based Probabilistic Approach. Els. Journal of App. Acoust. 70, 1168-1174. [PCA].

Lee, K.C., Ou, J.S., Huang, M.C., Fang, M.C., 2009b. A Novel Location Estimation based on Pattern Matching Algorithm in Underwater Environments. Els. Journal of App. Acoust. 70, 479-483. [PM].

Lee, K.C., Ou, J.S., Wang, L.T., 2009c. Underwater Acoustic Localization by Probabilistic Fingerprinting in Eigenspace. Proc. of the MTS/IEEE Oceans Biloxi [PF]. 
Luo, H., Guo, Z., Dong, W., 2010. LDB: Localization with Directional Beacons for Sparse 3D Underwater Acoustic Sensor Networks. Journal of Networks 5, 28-38. [LDB].

Luo, H., Zhao, Y., Guo, Z., Liu, S., Chen, P., Ni, L.M., 2008. UDB: Using Directional Beacons for Localization in Underwater Sensor Networks. Proc. of the ICPADS , 551-558[UDB].

Mackenzie, K.V., 1981. Nine-term Equation for Sound Speed in the Oceans. J. Acoust. Soc. Am. 70, 807-812.

Mirza, D., Schurgers, C., 2008. Motion-Aware Self-Localization for Underwater Networks. Proc. of the WUWNet, 51-58[MASL].

Molins, M., Stojanovic, M., 2006. Slotted FAMA: A MAC Protocol for Underwater Acoustic Networks. Proc. of MTS/IEEE OCEANS .

Moore, D., Leonard, J., Rus, D., Teller, S., 2004. Robust Distributed Network Localization with Noisy Range Measurement. Proc. of ACM Sensys, 5061.

Niculescu, D., Nathi, B., 2001. Ad hoc Positioning System. Proc. of IEEE Globecom , 2926-2931.

Novikov, A., Bagtzoglou, A.C., 2006. Hydrodynamic Model of the Lower Hudson River Estuarine System and its Application for Water Quality Management. Water Resources Management 20, 257-276.

Othman, A.K., 2008. GPS-less Localization Protocol for Underwater Acous- 
tic Networks. Proc. of the IFIP Int'l Conf. Wireless and Optical Comm. Networks [GPS-less].

Rowan, E., 2008. LBL Underwater Positioning. Hydro International 12.

Saleh, A.A., Valenzuela, R.A., 1987. A Statistical Model for Indoor Multipath Propagation. IEEE Journal on Selected Areas in Communications 5, $128-137$.

Stojanovic, M., 2003. Acoustic (Underwater) Communication. Encyclopedia of Communications, John Wiley and Sons.

Stojanovic, M., 2006. On the Relationship Between Capacity and Distance in an Underwater Acoustic Communication Channel. Proc. of the ACM International Workshop on UnderWater Networks (WUWNet) .

Syed, A., Heidemann, J., 2006. Time Synchronization for High Latency Acoustic Networks. Proc. of the IEEE INFOCOM , 1-12.

Tan, H.P., Eu, Z.A., Seah, W.K.G., 2009. An Enhanced Underwater Positioning System to Support Deepwater Installations. Proc. of the MTS/IEEE Oceans Biloxi [E-UPS].

Tan, H.P., Gabor, A.F., Eu, Z.A., Seah, W.K.G., 2010. A Wide Coverage Positioning System (WPS) for Underwater Localization. Proc. of IEEE ICC [WPS].

Thomas, H., 1998. Gib buoys: An interface between space and depths of the ocean, in: Proceedings of IEEE Autonomous Underwater Vehicles Conference, Cambridge, MA, USA. pp. 181-184. 
Vickery, K., 1998. Acoustic Positioning Systems - a practical overview of current systems. Proc. of the Dynamic Positioning Conference .

Zhou, Y., He, J., Chen, K., Chen, J., Liang, A., 2009. An Area Localization Scheme for Large Scale Underwater Wireless Sensor Networks. Proc. of the IEEE Int'l Conf. on Comm. and Mobile Computing , 543-547[3DMALS].

Zhou, Z., Cui, J.H., Bagtzoglou, A., 2008. Scalable Localization with Mobility Prediction for Underwater Sensor Networks. Proc. of the IEEE INFOCOM , 2198-2206[SLMP].

Zhou, Z., Cui, J.H., Zhou, S., 2010. Efficient Localization for Large-Scale Underwater Sensor Networks. Ad Hoc Networks 8, 267-279. [LSL]. 


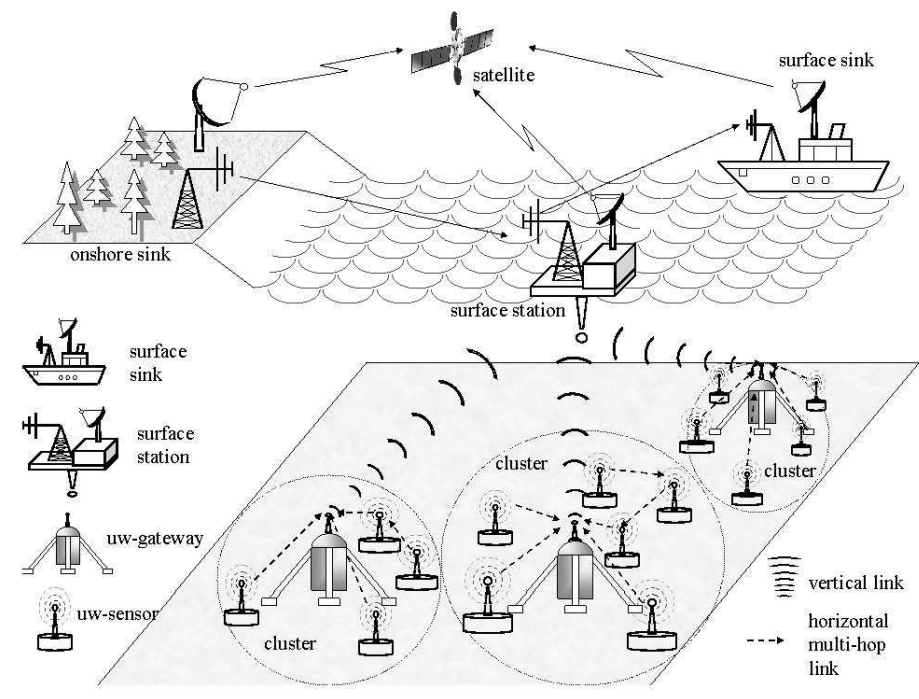

(a) 2D Network

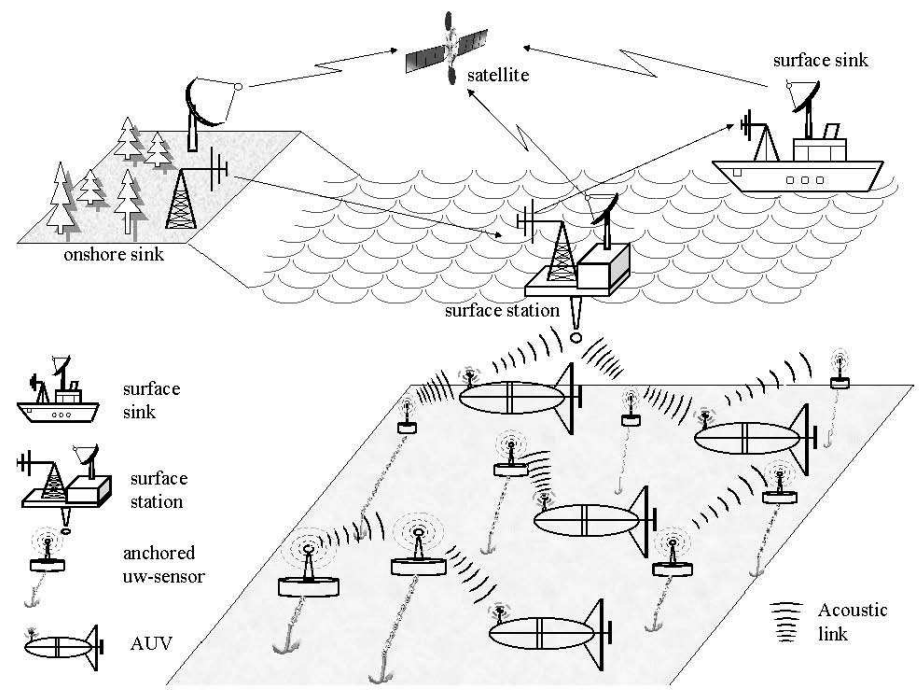

(b) 3D Network

Figure 1: Communications architecture for UWSNs (Akyildiz et al., 2007). 

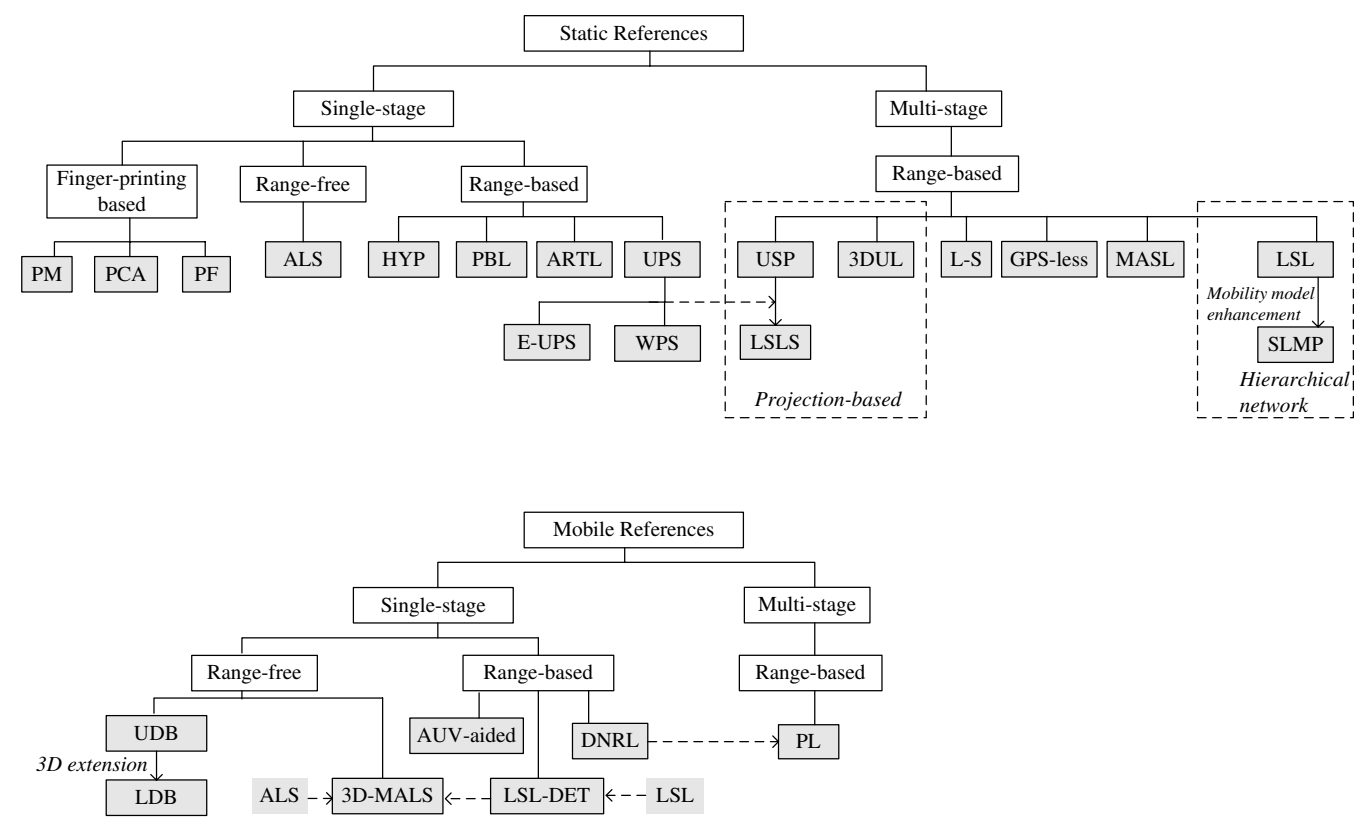

Figure 2: Classification of Localization schemes for Underwater Wireless Sensor Networks (UWSN). 


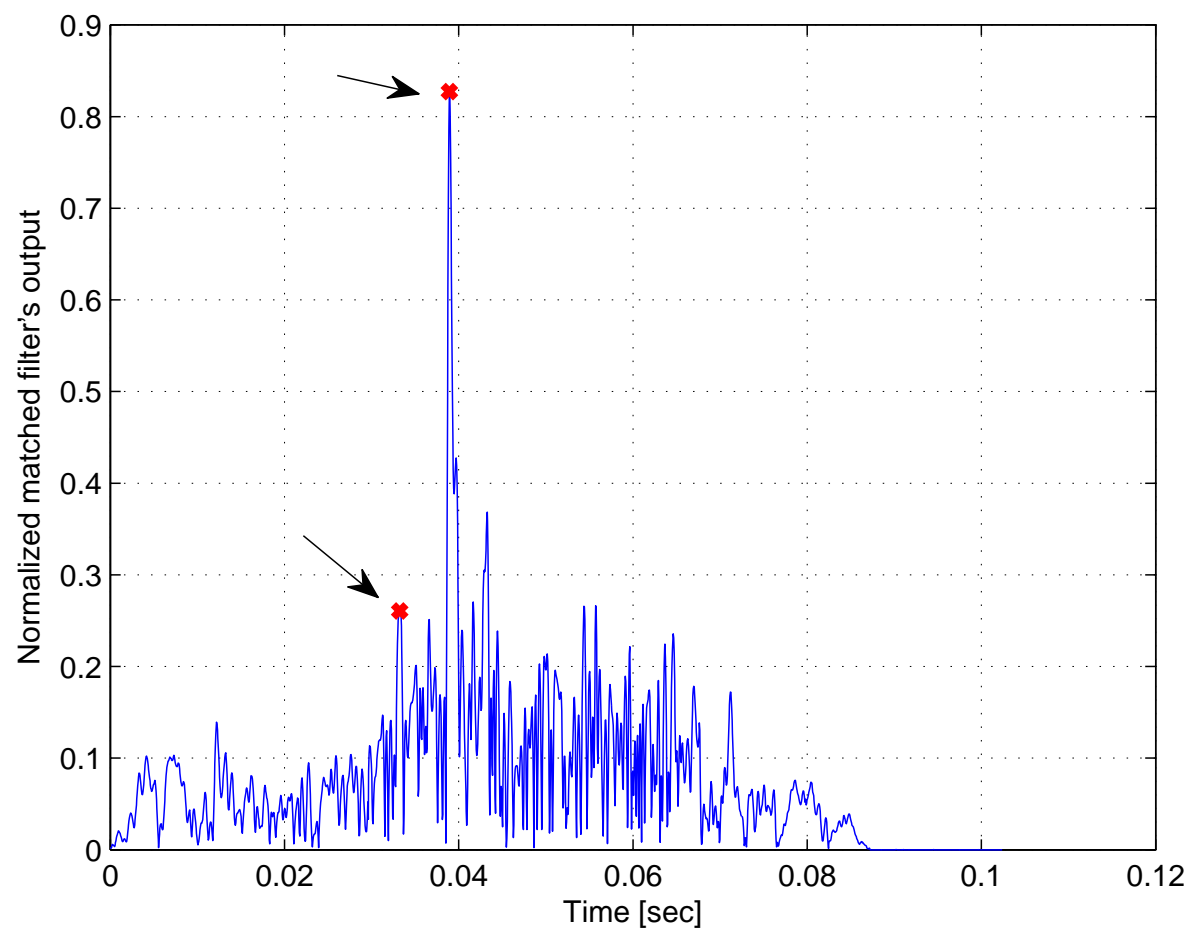

Figure 3: Example of matched filter output to illustrate that direct path signal may not be the strongest (Diamant and Horev, 2005). 


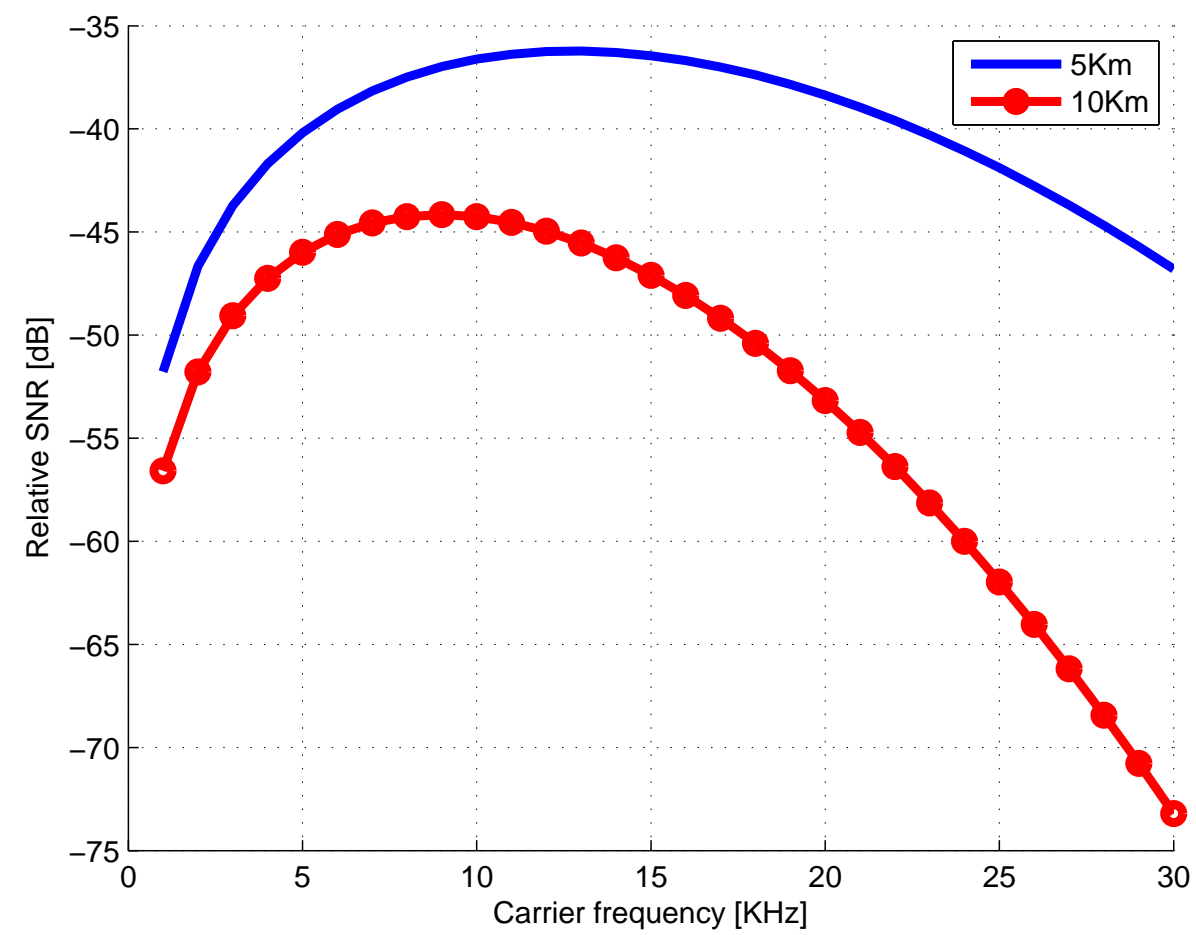

Figure 4: Relative SNR vs. carrier frequency and transmission distance. $\lambda=1.5$ and $\alpha$ calculated for $50 \mathrm{~m}$ depth, salinity of $38.8 \mathrm{ppt}$, and a temperature of $298.15 \mathrm{~K}$. 


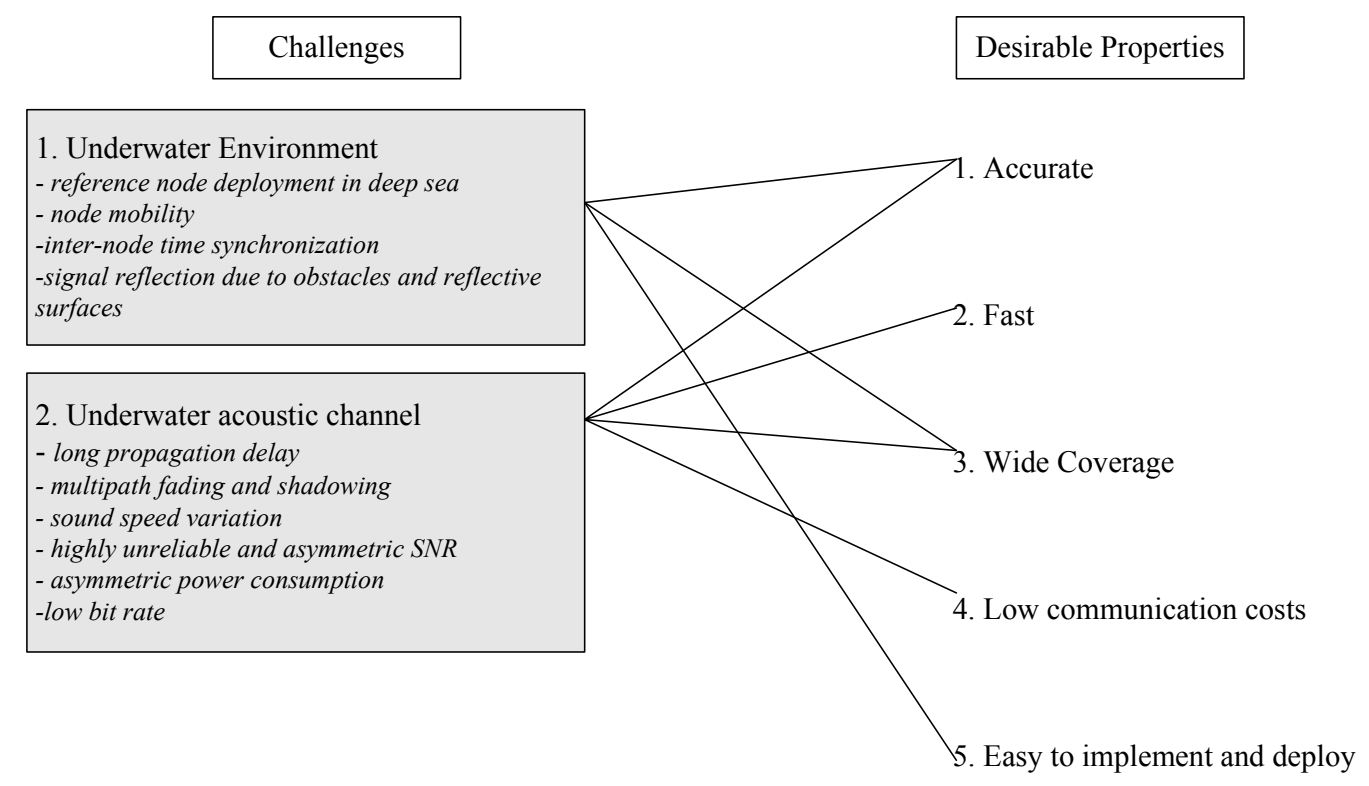

Figure 5: Mapping between the challenges and desirable performance of underwater localization. 


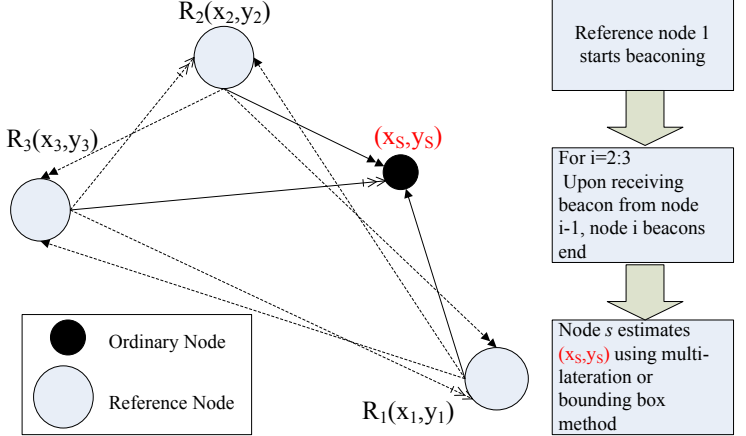

(a) Reactive beaconing mechanism of UPS

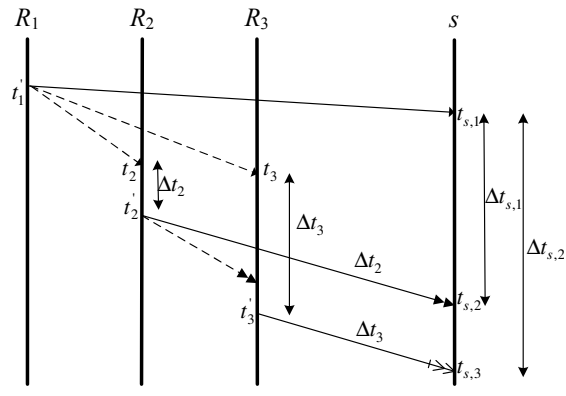

(b) Timing diagram of UPS

Figure 6: Illustration of (a) reactive beaconing mechanism and (b) timing diagram of UPS (2D). 


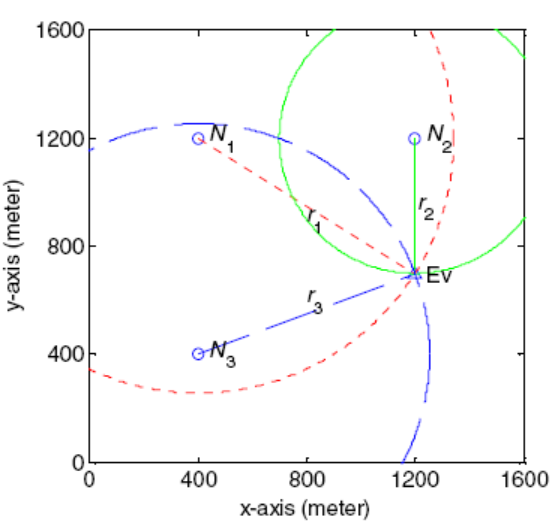

(a) circle-based approach

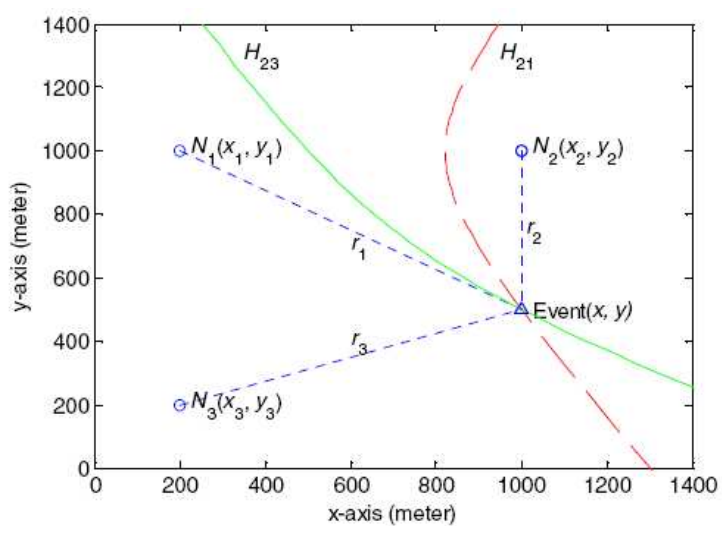

(b) hyperbola-based approach

Figure 7: Illustration of location estimation using (a) circle-based and (b) hyperbola-based approaches (Bian et al., 2009). 
Reference Node $R_{i}$ :

1. broadcast $i,\left(x_{i}, y_{i}, z_{i}\right), \eta$

Ordinary node $S$ :

1. broadcast $l_{i},\left(x_{i}, y_{i}, z_{i}\right)$ for all

$R_{i} \mathrm{U} N_{i}$

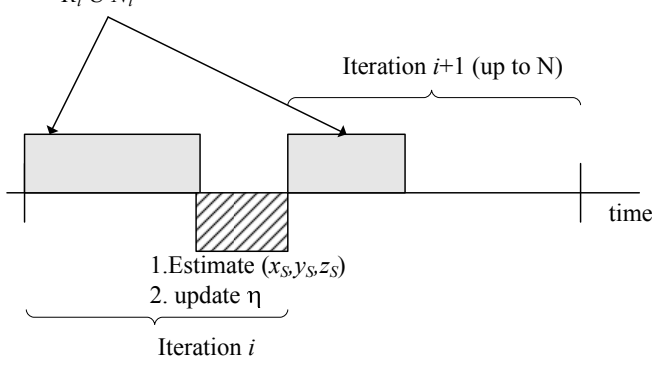

(a) Timing diagram for messaging in LSL and SLMP

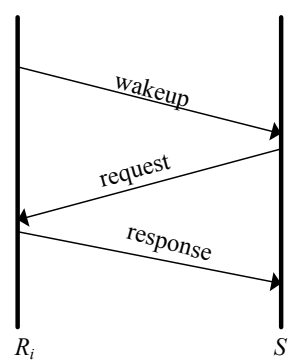

(b) Two-way messaging for 3DUL and AUV-aided localization

Figure 8: (a) Timing diagram of LSL and SLMP and (b) two-way message exchange for 3DUL and AUV-aided localization. 


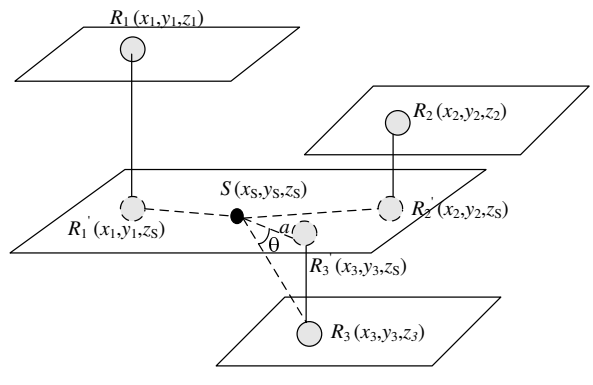

(a) Projection Mechanism in USP and 3DUL

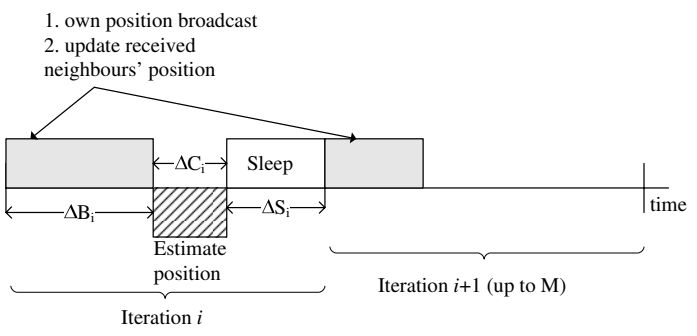

(b) Timing diagram for USP

Figure 9: (a) Projection mechanism in USP and 3DUL and (b) Timing diagram of USP. 


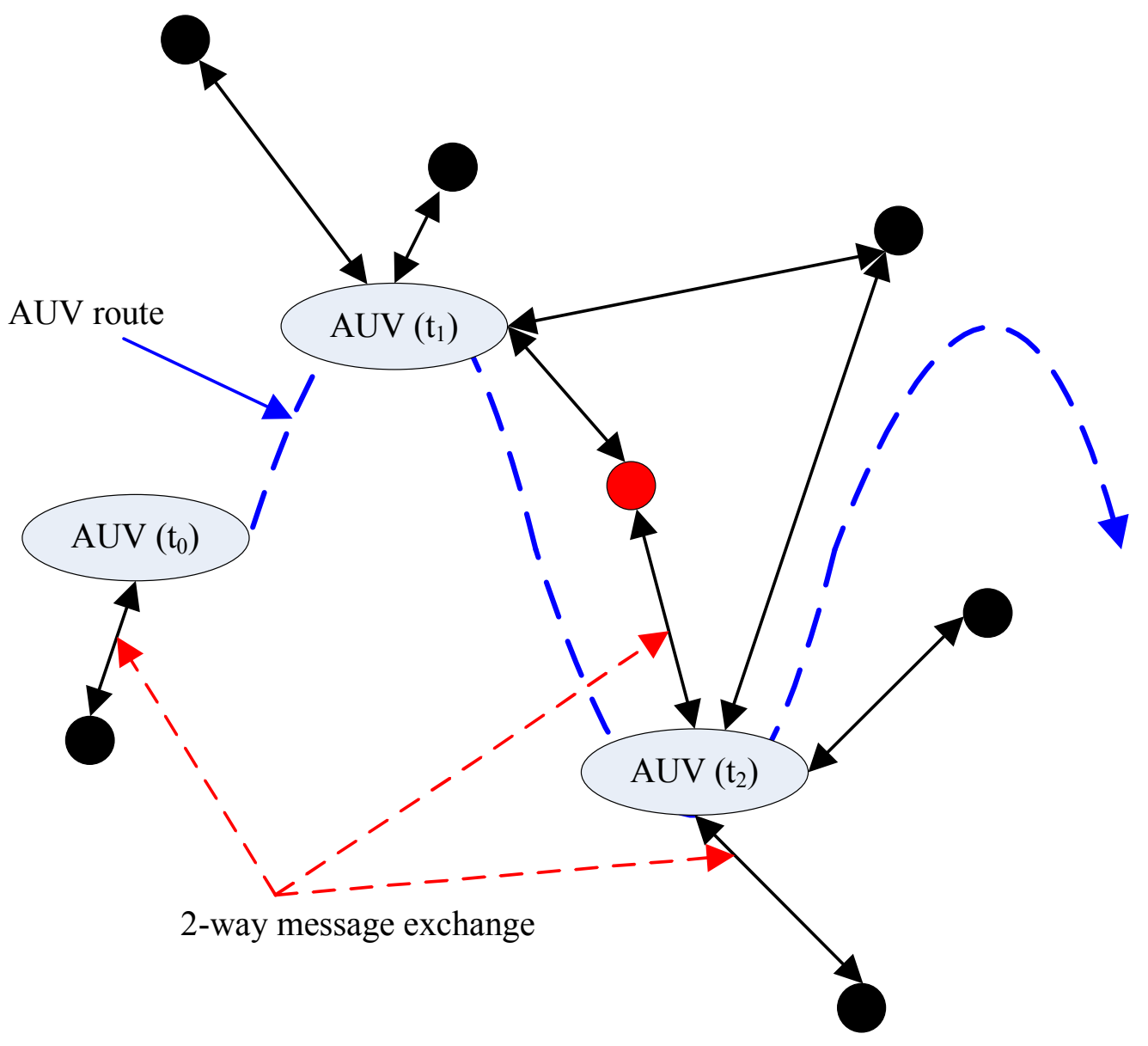

Figure 10: Illustration of AUV-Aided localization. 


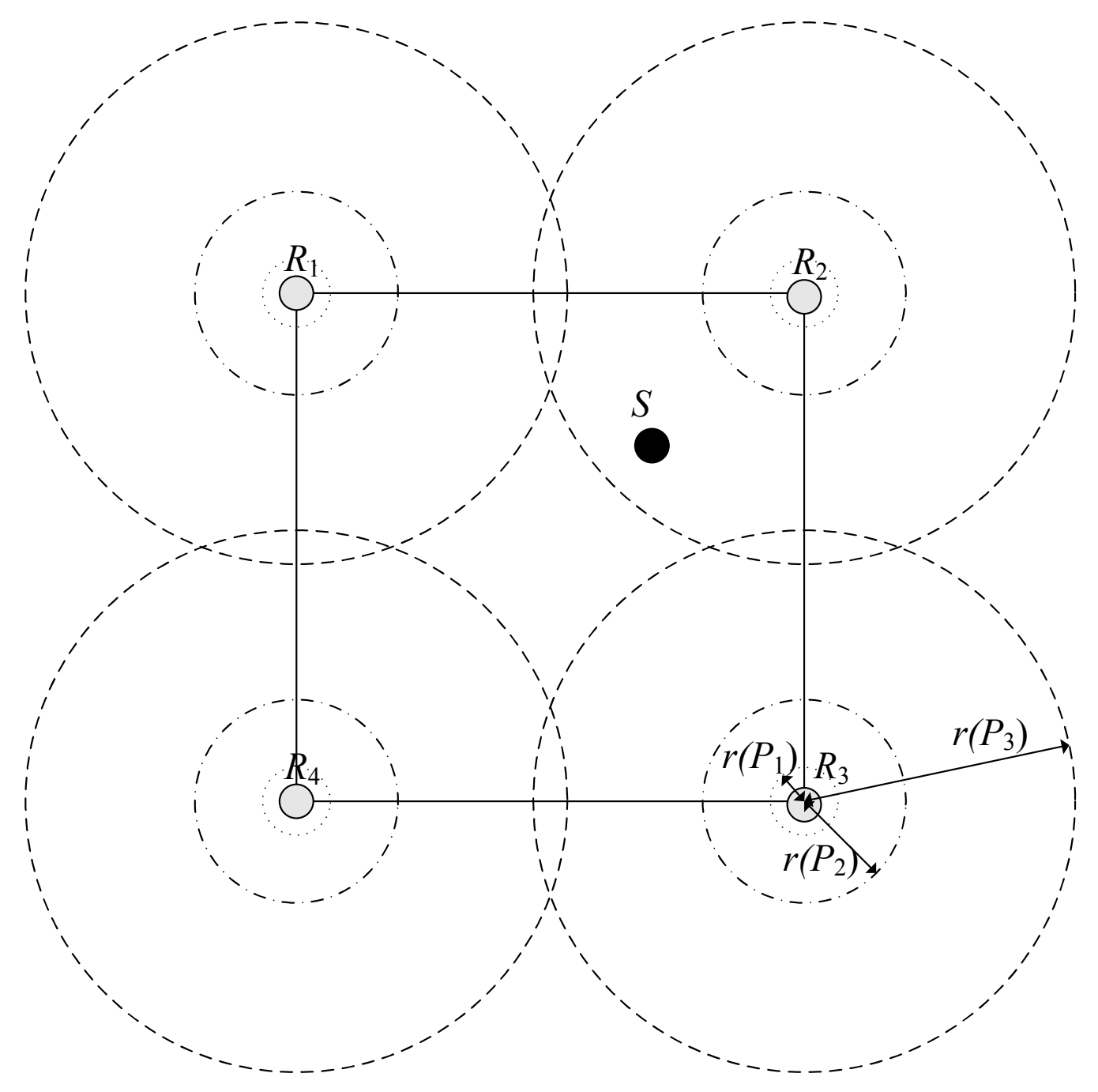

Figure 11: Illustration of 2D Area Localization Scheme (ALS). 

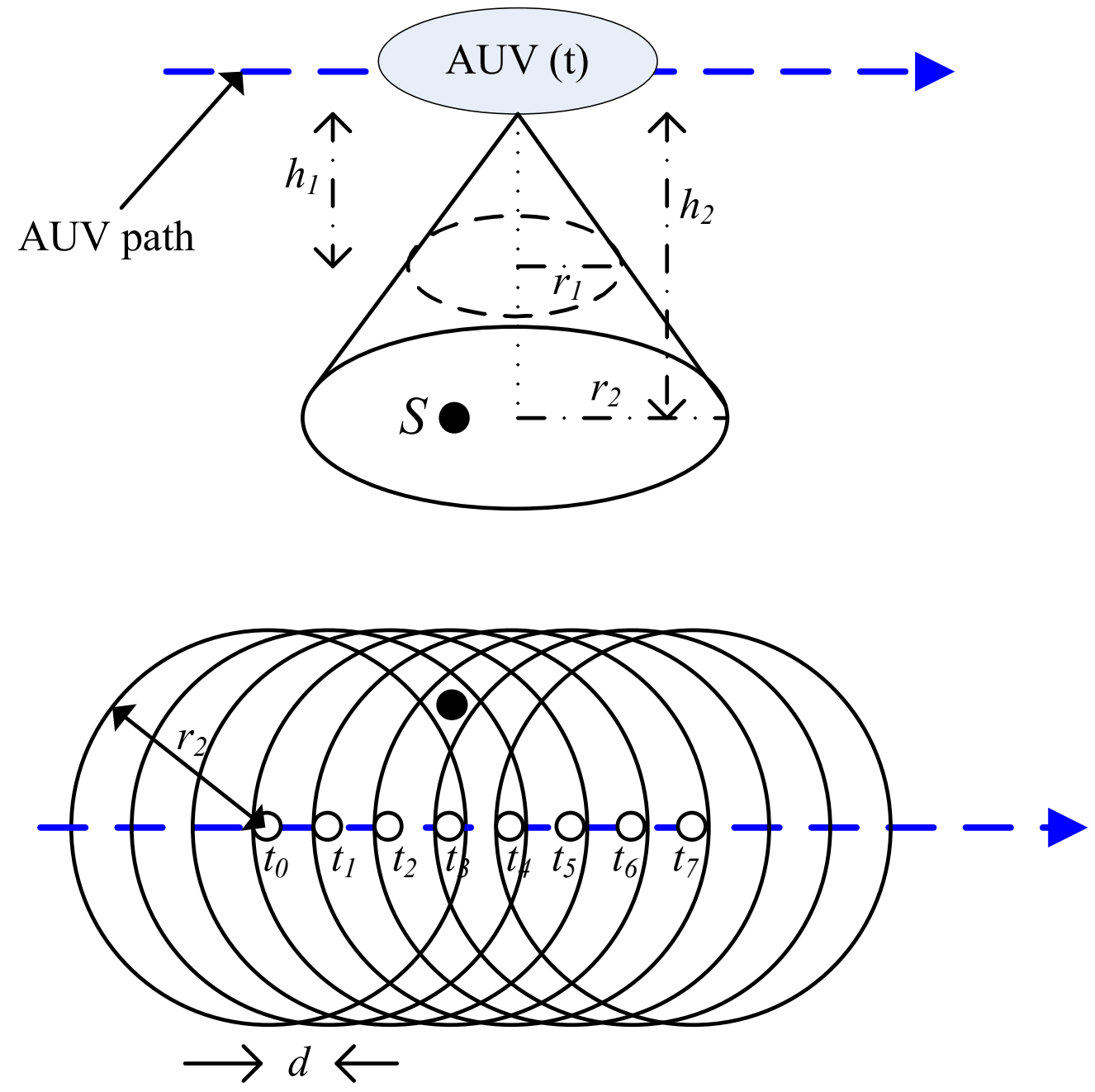

Figure 12: Illustration of Localization with Directional Beaconing (LDB). 


\begin{tabular}{|c|c|c|c|c|c|c|c|c|c|}
\hline \multirow[t]{3}{*}{ No. } & \multirow[t]{3}{*}{ Scheme } & \multirow[t]{3}{*}{ Remarks } & \multicolumn{7}{|c|}{ Challenges addressed } \\
\hline & & & \multicolumn{4}{|c|}{ Underwater Environment } & \multicolumn{3}{|c|}{ Underwater acoustic propagation } \\
\hline & & & $\begin{array}{l}\text { Infrastructure / } \\
\text { initial } \\
\text { reference } \\
\text { nodes needed }\end{array}$ & $\begin{array}{l}\text { Node } \\
\text { Mobility }\end{array}$ & $\begin{array}{l}\text { No inter- } \\
\text { node time } \\
\text { sync needed }\end{array}$ & NLOS & $\begin{array}{l}\text { Sound } \\
\text { speed } \\
\text { variation }\end{array}$ & $\begin{array}{l}\text { Transmission } \\
\text { losses }\end{array}$ & \begin{tabular}{|l|} 
Silent \\
Positioning
\end{tabular} \\
\hline \multicolumn{10}{|c|}{ Static References } \\
\hline 1 & UPS & Requires seabed reference & \multirow{5}{*}{$\begin{array}{l}4 \text { (including } 1 \\
\text { underwater) }\end{array}$} & & $\checkmark$ & $\checkmark$ & & & $\checkmark$ \\
\hline 2 & E-UPS & $\begin{array}{l}\text { Improves performance of UPS in harsh channel } \\
\text { environment }\end{array}$ & & & $\checkmark$ & & & $\checkmark$ & $\checkmark$ \\
\hline 3 & WPS & $\begin{array}{l}\text { Improve localization uniqueness of UPS with } \\
\text { additional reference }\end{array}$ & & & $\checkmark$ & & & $\checkmark$ & $\checkmark$ \\
\hline 4 & HYP & $\begin{array}{l}\text { Hyperbola-based approach to improve likelihood of } \\
\text { location estimation over circle-based approaches }\end{array}$ & & & $\checkmark$ & & & & $\checkmark$ \\
\hline 5 & PBL & $\begin{array}{l}\text { Uses probability models for measurement noise to } \\
\text { improve localization accuracy }\end{array}$ & & & $\checkmark$ & & & & $\checkmark$ \\
\hline 6 & UPS & $\begin{array}{l}\text { Projection-based approach to map 3D localization } \\
\text { into } 2 \mathrm{D} \text { problem }\end{array}$ & \multirow{3}{*}{$\begin{array}{l}3 \text { surface } \\
\text { references only }\end{array}$} & & & & & & $\checkmark$ \\
\hline 7 & 3DUL & $\begin{array}{l}\text { Improves on USP in terms of (i) sound speed } \\
\text { estimation and (ii) by not requiring inter-node time } \\
\text { sync through 2-way messaging }\end{array}$ & & $\checkmark$ & $\checkmark$ & & $\checkmark$ & & \\
\hline 8 & LSLS & Improves on USP with desirable properties of UPS & & & 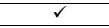 & & $\checkmark$ & & $\checkmark$ \\
\hline 9 & LSL & $\begin{array}{l}\text { Hierarchical localization that considers simple } \\
\text { mobility model in the performance evaluation }\end{array}$ & \multirow{2}{*}{$\begin{array}{l}\text { Surface buoys } \\
\text { and submerged } \\
\text { anchors }\end{array}$} & $\checkmark$ & & & & & $\checkmark$ \\
\hline 10 & SLMP & $\begin{array}{l}\text { Improves LSL with predictable mobility pattern in } \\
\text { sea shore environment }\end{array}$ & & $\checkmark$ & & & & & $\checkmark$ \\
\hline 11 & L-S & Performs joint localization and synchronization & 5 & & $\checkmark$ & & & & $\checkmark$ \\
\hline 12 & $\begin{array}{l}\text { GPS- } \\
\text { less }\end{array}$ & $\begin{array}{l}\text { 2D localization with respect to single initial } \\
\text { reference }\end{array}$ & 1 & & & & & & $\checkmark$ \\
\hline 13 & MASL & $\begin{array}{l}\text { Accounts for measurement errors due to node } \\
\text { mobility during localization epoch }\end{array}$ & $\begin{array}{l}4 \text { (including } 1 \\
\text { underwater) }\end{array}$ & $\checkmark$ & & & & & $\checkmark$ \\
\hline \multicolumn{10}{|c|}{ Mobile References } \\
\hline 14 & $\begin{array}{l}\text { AUV- } \\
\text { Aided }\end{array}$ & $\begin{array}{l}\text { Single AUV uses 2-way messaging and assumed its } \\
\text { position underwater is known accurately }\end{array}$ & $1 \mathrm{AUV}$ & & $\checkmark$ & & & & \\
\hline 15 & DNRL & $\begin{array}{l}\text { Dive-and-Rise beacons with meandering current } \\
\text { node mobility model }\end{array}$ & \multirow{2}{*}{$\begin{array}{l}\text { Surface buoys } \\
\text { with DNR } \\
\text { mechanism }\end{array}$} & $\checkmark$ & & & & & $\checkmark$ \\
\hline 16 & PL & Multi-stage DNRL & & $\checkmark$ & & & & & $\checkmark$ \\
\hline 17 & $\begin{array}{l}\text { LSL- } \\
\text { DET }\end{array}$ & $\begin{array}{l}\text { LSL with DNR using Detachable Elevator } \\
\text { Transducers }\end{array}$ & $\begin{array}{l}\text { Surface buoys } \\
\text { with DETs }\end{array}$ & & & & & & $\checkmark$ \\
\hline
\end{tabular}

Table 1: Extent to which range-based schemes have addressed challenges of underwater localization. 


\begin{tabular}{|l|l|l|l|}
\hline \multirow{2}{*}{ S/No } & \multirow{2}{*}{ Scheme } & Mechanism to control error propagation in multi-stage localization schemes \\
\cline { 3 - 4 } & & Criteria for 'new' reference node qualification & Selection of 'new' reference nodes for localization \\
\hline 1 & USP & Nil & Nil \\
\hline 2 & 3 DUL & Robust Virtual Anchors Plane & Nil (Reference nodes have finite lifetime) \\
\hline 3 & LSLS & Maximization of coverage & \multicolumn{2}{|l}{} \\
\hline 4 & LSL & Minimization of error (confidence threshold) & Time-stamp and confidence threshold \\
\hline 5 & SLMP & Minimization of error (confidence threshold) & Nil \\
\hline 6 & L-S & Nil & Maximization of coverage \\
\hline 7 & GPS-less & Nil & Nil \\
\hline 8 & MASL & Nil & Hop-count and time-stamp threshold \\
\hline 9 & PL & Depth criteria & \\
\hline
\end{tabular}

Table 2: Mechanism used by multi-stage schemes to trade-off between minimizing error propagation and delay while maximizing coverage and energy efficiency. 


\begin{tabular}{|l|l|l|l|l|l|l|l|l|}
\hline \multirow{2}{*}{ S/No } & Scheme & \multicolumn{2}{|l|}{ Simulation scenario } & \multicolumn{4}{l|}{ Localization performance } \\
\cline { 3 - 9 } & & Vol. $\left(\mathrm{m}^{3}\right)$ & No of nodes & No of initial reference nodes & Comm. Costs. & Coverage & time & Accuracy \\
\hline 1 & USP & $100 \times 100 \times 100$ & 1000 & 3 surface & 3 msg/node & $28 \%$ & 35 iterations & 0.43 \\
\hline 2 & LSLS & $100 \times 100 \times 200$ & 2000 & 3 surface & $5 \%$ active & $5 \%$ & & 0.25 \\
\hline 3 & 3DUL & $100 \times 100 \times 100$ & 500 & 3 surface & 8 msg/node & $44 \%$ & & 0.03 \\
\hline 4 & LSL & $1000 \times 1000 \times 600$ & 250 & 25 & $60 /$ node & $80 \%$ & $3000 \mathrm{sec}$ & 0.4 \\
\hline 5 & SLMP & $100 \times 100 \times 100$ & 500 & 50 & 8 msg/node & $30 \%$ & & 0.16 \\
\hline 6 & AUV-aided & $1000 \times 1000 \times 120$ & 150 & 1 & $70 \mathrm{msg} / \mathrm{node}$ & $40 \%$ & $3600 \mathrm{sec}$ & 0.167 \\
\hline 7 & DNRL & $1000 \times 1000 \times 600$ & 250 & 25 & $5 \mathrm{msg} / \mathrm{node}$ & $80 \%$ & $3500 \mathrm{sec}$ & 0.23 \\
\hline 8 & PL & $1000 \times 1000 \times 600$ & 250 & 25 & $8 \mathrm{msg} / \mathrm{node}$ & $80 \%$ & $2500 \mathrm{sec}$ & 0.6 \\
\hline 9 & LSL-DET & $1000 \times 1000 \times 1000$ & 250 & 37 & & $57 \%$ & & 0.0167 \\
\hline
\end{tabular}

Table 3: Comparative performance of range-based underwater localization schemes. 